\title{
Singular Optimal Controls of Stochastic Recursive Systems and Hamilton-Jacobi-Bellman Inequality
}

\author{
Liangquan Zhang ${ }^{1}$ \\ 1. School of Science, \\ Beijing University of Posts and Telecommunications, \\ Beijing 100876, China
}

\begin{abstract}
In this paper, we study the optimal singular controls for stochastic recursive systems, in which the control has two components: the regular control, and the singular control. Under certain assumptions, we establish the dynamic programming principle for this kind of optimal singular controls problem, and prove that the value function is a unique viscosity solution of the corresponding Hamilton-Jacobi-Bellman inequality, in a given class of bounded and continuous functions. At last, an example is given for illustration.
\end{abstract}

Keywords: Dynamic programming principle (DPP in short), Forward-backward stochastic differential equations (FBSDEs in short), Hamilton-Jacobi-Bellman inequality (H-J-B inequality in short), Optimal control, Singular Control, Value function, Viscosity solution.

2000 MSC: 93E20, 60H15, 60H30

\footnotetext{
${ }^{1}$ The author acknowledges the financial support partly by the National Nature Science Foundation of China (Grant No. 11701040, 61571059, 11871010 \& 61603049) and Innovation Foundation of BUPT for Youth (No. 500417024 \& 505018304). E-mail: xiaoquan51011@163.com
} 


\section{Introduction}

Singular stochastic control problem is one of the most important topics in fields of stochastic control. This problem was first introduced by Bather and Chernoff [11] in 1967 by considering a simplified model for the control of a spaceship. It was noted for this special model that there was a connection between the singular control problem and optimal stopping problem. This link was established through the derivative of the value function of this initial singular control problem and the value function of the corresponding optimal stopping problem. From then on, it was extended by Beněs, Shepp, Witzsenhausen (see [3]) and Karatzas and Shreve (see [39, 40, 41, 42, 43]). In contrast to classical control problems, singular control problems admit both of the continuity of the cumulative displacement of the state caused by control and the jump of one in impulsive control problems, between which it is either constant or absolutely continuous.

Recall the classical singular control problems: The state process is driven by the following $n$-dimensional stochastic differential equation

$$
\left\{\begin{aligned}
\mathrm{d} X_{s}^{t, x ; v, \xi} & =b\left(s, X_{s}^{t, x ; v, \xi}, v_{s}\right) \mathrm{d} s+\sigma\left(s, X_{s}^{t, x ; v, \xi}, v_{s}\right) \mathrm{d} W_{s}+G_{s} \mathrm{~d} \xi_{s} \\
X_{t}^{t, x ; v, \xi} & =x, \quad 0 \leq t \leq s \leq T
\end{aligned}\right.
$$

on some filtered probability space $(\Omega, \mathscr{F}, P)$, where $b(\cdot, \cdot, \cdot):[0, T] \times \mathbb{R}^{n} \times$ $\mathbb{R}^{k} \rightarrow \mathbb{R}^{n}, \sigma(\cdot, \cdot, \cdot):[0, T] \times \mathbb{R}^{n} \times \mathbb{R}^{k} \rightarrow \mathbb{R}^{n \times d}, G(\cdot):[0, T] \rightarrow \mathbb{R}^{n \times m}$ are given deterministic functions, $\left(W_{s}\right)_{s \geq 0}$ is an $d$-dimensional Brownian motion, $(x, t)$ are initial time and state, $v(\cdot):[0, T] \rightarrow \mathbb{R}^{k}$ is classical control process, and $\xi(\cdot):[0, T] \rightarrow \mathbb{R}^{m}$, with nondecreasing left-continuous with right limits stands for the singular control.

The aim is to minimize the cost functional:

$$
J(t, x ; v, \xi)=\mathbb{E}\left[\int_{t}^{T} l\left(s, X_{s}^{t, x ; v, \xi}, v_{s}\right) \mathrm{d} s+\int_{t}^{T} K_{s} \mathrm{~d} \xi_{s}\right]
$$

where

$$
\begin{aligned}
l(\cdot, \cdot, \cdot) & :[0, T] \times \mathbb{R}^{n} \times \mathbb{R}^{k} \rightarrow \mathbb{R} \\
K(\cdot) & :[0, T] \rightarrow \mathbb{R}_{+}^{m} \triangleq\left\{x \in \mathbb{R}^{m}: x_{i} \geq 0, i=1, \ldots m\right\}
\end{aligned}
$$

are given deterministic functions, where $l(\cdot)$ represents the running cost tare of the problem and $K(\cdot)$ the cost rate of applying the singular control. 
There are four approaches employed: the first one is based on the theory of partial differential equations and on variational arguments, and can be found in the works of Alvarez [1, 2], Chow, Menaldi, and Robin [19], Karatzas [40], Karatzas and Shreve [43], and Menaldi and Taksar [52]. The second one is related to probabilistic methods; see Baldursson [6]. Boetius [8, 9], Boetius and Kohlmann [10], El Karoui and Karatzas [29, 30], Karatzas [39], and Karatzas and Shreve [41, 42]. Third, the DPP, has been studied in a general context, for example, by Boetius [9], Haussmann and Suo [36], Fleming and Soner [31], and Zhu [66]. At last the maximum principle for optimal singular controls (see, for example, Cadenillas and Haussmann [17], Dufour and Miller [26], Dahl and Øksendal [27] see references therein). The existence for optimal singular control can be found in Haussmann and Suo [36] and Dufour and Miller [25] via different approaches.

It is necessary to point out that singular control problems are largely used in diverse areas such as mathematical finance (see Baldursson and Karatzas [12], Chiarolla and Haussmann [18], Kobila [44], and Karatzas and Wang [45], Davis and Norman [24]), manufacturing systems (see, Shreve, Lehoczky, and Gaver [60]), and queuing systems (see Martins and Kushner [53]). Particularly, the application of H-J-B inequality in finance can be seen in Pagès and Possamaï [58], which is employed to investigate the bank monitoring incentives.

As is well known for the classical stochastic control problems, DPP is satisfied. Moreover, if the value function has appropriate regularity, it admits a second-order nonlinear partial differential equation (H-J-B equation) (see Fleming and Rishel [32] and Lions [49] reference therein). In the frame work of singular stochastic control, the H-J-B equation becomes a second-order variational inequality (see Fleming and Soner [31], Haussmannand, Suo [35]).

Wang [63] firstly introduces and studies a class of singular control problems with recursive utility, where the cost function is determined by a backward stochastic differential equation (BSDE in short). More preciously, the cost functional is defined by

$$
J(t, x ; \xi)=Y_{t}^{t, x ; \xi},
$$

where $Y_{t}^{t, x ; v, \xi}$ is determined by

$$
\left\{\begin{aligned}
\mathrm{d} X_{s}^{t, x ; \xi} & =\left(a X_{s}^{t, x ; \xi}+b\right) \mathrm{d} s+\sigma \mathrm{d} W_{s}+\mathrm{d} \xi_{s} \\
\mathrm{~d} Y_{s}^{t, x ; \xi} & =-f\left(Y_{s}^{t, x ; \xi}, Z_{s}^{t, x ; \xi}, v_{s}\right)-\mu Z_{s}^{t, x ; \xi} \mathrm{d} s+Z_{s}^{t, x ; \xi} \mathrm{d} W_{s}-\mathrm{d} \xi_{s}, \\
X_{t}^{t, x ; \xi} & =x, Y_{T}^{t, x ; \xi}=0, \quad 0 \leq t \leq s \leq T .
\end{aligned}\right.
$$


The value function is defined by

$$
u(t, x)=\inf _{\xi \in \mathcal{V}} J(t, x ; \xi),
$$

where $\mathcal{V}$ denotes the set of admissible controls $\xi(\cdot)$ which are $\mathscr{F}_{t}$-adapted processes from $[0, T]$ into $\mathbb{R}$, left continuous having right limits, nonnegative and nondecreasing, moreover $\xi_{t}=0$. Under certain assumptions, the author proved that the value function is a nonnegative, convex solution of the $\mathrm{H}-\mathrm{J}-\mathrm{B}$ equation:

$$
\min \left\{\frac{\partial}{\partial t} u+\frac{1}{2} \sigma^{2} \frac{\partial^{2}}{\partial x^{2}} u+(a x+b) \frac{\partial}{\partial x} u, u_{x}+1\right\}=0, u(T, x)=0 .
$$

As a matter of fact, the class of stochastic control problems with recursive utility was firstly considered by Peng [56] where he considered an absolutely continuous stochastic control problem with recursive utility, in which the cost function is defined by a BSDE. As is well known, BSDE has been widely used in mathematical finance and differential games, partial differential equation, etc (see [38, 55, 56, 34, 64, 47]). Since then, there exists a huge literature to focus on the stochastic recursive optimal control problems (see reference therein).

Motivated by above work, in this paper, we consider singular controls problem of a general framework like:

$$
\left\{\begin{array}{l}
\mathrm{d} X_{s}^{t, x ; v, \xi}=b\left(s, X_{s}^{t, x ; v, \xi}, v_{s}\right) \mathrm{d} s+\sigma\left(s, X_{s}^{t, x ; v, \xi}, v_{s}\right) \mathrm{d} W_{s}+G \mathrm{~d} \xi_{s}, \\
\mathrm{~d} Y_{s}^{t, x ; v, \xi}=-f\left(t, X_{s}^{t, x ; v, \xi}, Y_{s}^{t, x ; v, \xi}, Z_{s}^{t, x ; v, \xi}, v_{s}\right) \mathrm{d} s+Z_{s}^{t, x ; v, \xi} \mathrm{d} W_{s}-K \mathrm{~d} \xi_{s}, \\
X_{t}^{t, x ; v, \xi}=x, Y_{T}^{t, x ; v, \xi}=\Phi\left(X_{T}^{t, x ; v, \xi}\right), \quad 0 \leq t \leq s \leq T,
\end{array}\right.
$$

with the similar cost functional

$$
J(t, x ; \xi)=Y_{t}^{t, x ; v, \xi}
$$

Note that $G$ and $K$ are deterministic matrices in Eq. (5). The justification will be given in Remark 2 below. Using some properties of the BSDE and analysis technique, we expand the extension of the dynamic programming principle of the recursive control problem in [16, 22, 21, 50, 56, 61, 63, 68 to the singular controls case. And then, we show that, provided the problem is formulated within a Markovian framework, the value function is a unique viscosity solution of the problem for one kind of nonlinear H-J-B inequality, 
in a given class of bounded and continuous functions. Some characteristics of the value function of the control problem are obtained as well. A concrete example is provided to validate our theoretic results.

To the best of our knowledge, such singular optimal controls of FBSDEs (5) via DPP have not been studied before. On the one hand, our paper generalizes the DPP of the forward controls problem by Haussmann and Suo [36] to recursive situation, backward one in Peng [56, 57], Wu and $\mathrm{Yu}$ 64 to the singular control case, and admits the following advantages and improvements. First, in Peng [56, 57], $\mathrm{Wu}$ and $\mathrm{Yu}[64]$, the recursive cost function does not have the singular control. In our paper, the optimization problem has singular control for the cost function which is described by the solution of BSDE. In contrast to $\mathrm{Wu}$ and $\mathrm{Yu}$ [64], our variational inequality involves the derivative of the value function. As a matter of fact, Example 1.7 in the classical literature by Crandall, Ishii and Lions [20] displays such a kind of obstacle and gradient constraint problems. Thus, the purpose of this paper is to response this item and to deepen and enrich this research. Besides, through the value function, we can define the so called inaction region, in which the status process is continuous and the optimal singular control should not produce any jump. Our ideas and methodology of proof in our paper for DPP come from Haussmannand, Suo [36], Peng [56, 57], $\mathrm{Wu}$ and $\mathrm{Yu}$ [64]. Second, the method of proof in our paper is mainly based on elementary mathematics analysis technique and the properties of BSDE. Wang [63] studies a particular situation. However, the FBSDEs in Wang [63] do not contain the regular control and the generator is not general case. The solution there belongs to Sobolev space. Nonetheless, in classical stochastic control theory, viscosity solution is usually employed to study H-J-B equation. In this paper, we adopt the approach developed by Fleming and Soner [31] to get the uniqueness proof for the viscosity solution of corresponding H-J-B inequality.

The rest of this paper is organized as follows: After some preliminaries in Section 2, we are devoted the third section to the DPP for the optimal singular controls problem and prove that the value function of the singular controls problem is the unique viscosity solution of the corresponding H-J-B inequality. We conclude with a concrete example in Section 4. Finally, some proofs of lemmas are scheduled in the Appendix. 


\section{Formulation of the Problem}

Throughout this paper, we denote by $\mathbb{R}^{n}$ the space of $n$-dimensional Euclidean space, by $\mathbb{R}^{n \times d}$ the space the matrices with order $n \times d$. The matrix transpose is denoted by $T$. Let $\left(\Omega, \mathscr{F},\left\{\mathscr{F}_{t}\right\}_{t \geq 0}, P\right)$ be a complete filtered probability space on which a $d$-dimensional standard Brownian motion $W(\cdot)$ is defined, with $\left\{\mathscr{F}_{t}\right\}_{t \geq 0}$ being its natural filtration, augmented by all the $P$-null sets. Given a subset $U$ (compact) of $\mathbb{R}^{k}$, we will denote $\mathcal{U}$ the class of measurable, adapted processes $(v, \xi):[0, T] \times \Omega \rightarrow U \times[0, \infty)^{m}$, with $\xi$ nondecreasing left-continuous with right limits and $\xi_{0}=0$, moreover, $\mathbb{E}\left[\sup _{0 \leq t \leq T}\left|v_{t}\right|^{2}+\left|\xi_{T}\right|^{2}\right]<+\infty$. For each $t>0$, we denote by $\left\{\mathscr{F}_{s}^{t}, t \leq s \leq T\right\}$ the natural filtration of the Brownian motion $\left\{W_{s}-W_{t}, t \leq s \leq T\right\}$, augmented by the $P$-null sets of $\mathscr{F}$. In what follows, $C$ represents a generic constant, which can be different from line to line.

We now introduce the following spaces of processes:

$$
\begin{aligned}
& \mathcal{S}^{2}(0, T ; \mathbb{R}) \triangleq\left\{\mathbb{R}^{n} \text {-valued } \mathscr{F}_{t^{-}} \text {adapted process } \phi(t) ; \mathbb{E}\left[\sup _{0 \leq t \leq T}\left|\phi_{t}\right|^{2}\right]<\infty\right\} \\
& \mathcal{M}^{2}(0, T ; \mathbb{R}) \triangleq\left\{\mathbb{R}^{n} \text {-valued } \mathscr{F}_{t^{-}} \text {adapted process } \varphi(t) ; \mathbb{E}\left[\int_{0}^{T}\left|\varphi_{t}\right|^{2} \mathrm{~d} t\right]<\infty\right\}
\end{aligned}
$$

and denote $\mathcal{N}^{2}[0, T]=\mathcal{S}^{2}\left(0, T ; \mathbb{R}^{n}\right) \times \mathcal{S}^{2}(0, T ; \mathbb{R}) \times \mathcal{M}^{2}\left(0, T ; \mathbb{R}^{n}\right)$. Clearly, $\mathcal{N}^{2}[0, T]$ forms a Banach space.

For any $v(\cdot) \times \xi(\cdot) \in \mathcal{U}$, we study the stochastic control systems governed by FBSDE of the following type with two adapted control processes:

$$
\left\{\begin{aligned}
\mathrm{d} X_{s}^{t, x ; v, \xi} & =b\left(s, X_{s}^{t, x ; v, \xi}, v_{s}\right) \mathrm{d} s+\sigma\left(s, X_{s}^{t, x ; v, \xi}, v_{s}\right) \mathrm{d} W_{s}+G \mathrm{~d} \xi_{s}, \\
\mathrm{~d} Y_{s}^{t, x ; v, \xi} & =-f\left(s, X_{s}^{t, x ; v, \xi}, Y_{s}^{t, x ; v, \xi}, Z_{s}^{t, x ; v, \xi}, v_{s}\right) \mathrm{d} s+Z_{s}^{t, x ; v, \xi} \mathrm{d} W_{s}-K \mathrm{~d} \xi_{s}, \\
X_{t}^{t, x ; v, \xi} & =x, Y_{T}^{t, x ; v, \xi}=\Phi\left(X_{T}^{t, x ; v, \xi}\right), \quad 0 \leq t \leq s \leq T,
\end{aligned}\right.
$$

where

$$
\begin{aligned}
b & :[0, T] \times \mathbb{R}^{n} \times \mathbb{R}^{k} \rightarrow \mathbb{R}^{n}, \\
\sigma & :[0, T] \times \mathbb{R}^{n} \times \mathbb{R}^{k} \rightarrow \mathbb{R}^{n \times d} \\
f: & {[0, T] \times \mathbb{R}^{n} \times \mathbb{R} \times \mathbb{R}^{d} \times \mathbb{R}^{k} \rightarrow \mathbb{R} } \\
\Phi & : \mathbb{R}^{n} \rightarrow \mathbb{R} .
\end{aligned}
$$


Definition 1. For any $v(\cdot) \times \xi(\cdot) \in \mathcal{U}$, a triple of processes

$$
\left(X^{t, x ; v, \xi}, Y^{t, x ; v, \xi}, Z^{t, x ; v, \xi}\right):[0, T] \times \Omega \rightarrow \mathbb{R}^{n} \times \mathbb{R} \times \mathbb{R}^{d}
$$

is called an adapted solution of the FBSDEs (17), if $\left(X^{t, x ; v, \xi}, Y^{t, x ; v, \xi}, Z^{t, x ; v, \xi}\right) \in$ $\mathcal{N}^{2}[0, T]$, and it satisfies (7), P-almost surely.

For simplicity, we adopt the following assumptions taken from Peng [57].

(A1) $b, \sigma$ are uniformly continuous in $(s, x, u)$, and there exits a positive constant $C$ such that for all $s \in[0, T], x^{1}, x^{2} \in \mathbb{R}^{n}, u^{1}, u^{2} \in \mathbb{R}^{k}$,

$$
\begin{aligned}
& \left|b\left(s, x^{1}, u^{1}\right)-b\left(s, x^{2}, u^{2}\right)\right|+\left|\sigma\left(s, x^{1}, u^{1}\right)-\sigma\left(s, x^{2}, u^{2}\right)\right| \\
\leq & C\left(\left|x^{1}-x^{2}\right|+\left|u^{1}-u^{2}\right|\right),
\end{aligned}
$$

and

$$
|b(s, x, u)|+|\sigma(s, x, u)| \leq C(1+|x|) .
$$

(A2) $f, \Phi$ are uniformly continuous in $(s, x, y, u)$ and there exists a constant $C>0$ such that for all $s \in[0, T], x^{1}, x^{2} \in \mathbb{R}^{n}, y^{1}, y^{2} \in \mathbb{R}, z^{1}, z^{2} \in \mathbb{R}^{d}$, $u^{1}, u^{2} \in \mathbb{R}^{k}$,

$$
\begin{aligned}
& \left|f\left(s, x^{1}, y^{1}, z^{1}, u^{1}\right)-f\left(s, x^{2}, y^{2}, z^{2}, u^{2}\right)\right|+\left|\Phi\left(x^{1}\right)-\Phi\left(x^{2}\right)\right| \\
\leq & C\left(\left|x^{1}-x^{2}\right|+\left|y^{1}-y^{2}\right|+\left|z^{1}-z^{2}\right|+\left|u^{1}-u^{2}\right|\right),
\end{aligned}
$$

moreover,

$$
|f(s, x, 0,0, u)|+|\Phi(x)| \leq C(1+|x|) .
$$

(A3) $G_{n \times m}$ and $K_{1 \times m}$ are deterministic matrices. Moreover, postulate that there exists $k_{0}$ such that $K^{i}>k_{0}>0,1 \leq i \leq m$, where $K^{i}$ denotes the $i$ th coordinate of $K$.

Remark 2. In this paper, we assume that $G_{n \times m}$ and $K_{1 \times m}$ are deterministic matrices. On the one hand, from the derivations in Theorem 5.1 of [36], also in Lemma 15 below, in our paper, it is convenient to show the "inaction" region for singular control; On the other hand, we may regard $Y_{s}^{t, x ; v, \xi}+G \xi_{s}$ together as a solution, in this way, we are able to apply the classical Itô's formula, avoiding the appearance of jump. We believe these assumptions can be removed properly, but at present, we consider constant only in our paper for our aim. Whilst in order to get the uniqueness of the solution to H-J-B inequality (30), we add the assumption $K^{i}>k_{0}>0,1 \leq i \leq m$. More details, see Theorem 21 below. 
Under above assumptions (A1)-(A3), for any $v(\cdot) \times \xi(\cdot) \in \mathcal{U}$, it is easy to check that FBSDEs (17) admit a unique $\mathcal{F}_{t}$-adapted solution denoted by the triple $\left(X^{t, x ; v, \xi}, Y^{t, x ; v, \xi}, Z^{t, x ; v, \xi}\right) \in \mathcal{N}^{2}[0, T]$ (See Pardoux and Peng [54]).

Like Peng [57], given any control processes $v(\cdot) \times \xi(\cdot) \in \mathcal{U}$, we introduce the following cost functional:

$$
J(t, x ; v(\cdot), \xi(\cdot))=\left.Y_{s}^{t, x ; v, \xi}\right|_{s=t}, \quad(t, x) \in[0, T] \times \mathbb{R}^{n} .
$$

We are interested in the value function of the stochastic optimal control problem

$$
\begin{aligned}
u(t, x) & =J(t, x ; \hat{v}(\cdot), \hat{\xi}(\cdot)) \\
& =e s s \inf _{v(\cdot) \times \xi(\cdot) \in \mathcal{U}} J(t, x ; v(\cdot), \xi(\cdot)), \quad(t, x) \in[0, T] \times \mathbb{R}^{n} .
\end{aligned}
$$

Since the value function (9) is defined by the solution of controlled BSDE (7), so from the existence and uniqueness, $u$ is well-defined.

Any $\hat{v}(\cdot) \times \hat{\xi}(\cdot) \in \mathcal{U}$ satisfying (9) is called an optimal control pair of optimal singular problem, and the corresponding state processes, denoted by $\left(X^{t, x ; \hat{v}, \hat{\xi}}, Y^{t, x ; \hat{v}, \hat{\xi}}, Z^{t, x ; \hat{v}, \hat{\xi}}\right)$, is called optimal state process. We also refer to $\left(X^{t, x ; \hat{v}, \hat{\xi}}, Y^{t, x ; \hat{v}, \hat{\xi}}, Z^{t, x ; \hat{v}, \hat{\xi}}, \hat{v}(\cdot), \hat{\xi}(\cdot)\right)$ as an optimal 5-tuple of optimal singular problem.

Definition 3 (Optimal Control). Any admissible controls $\hat{u}(\cdot) \times \hat{\xi}(\cdot) \in \mathcal{U}$, are called optimal, if $\hat{u}(\cdot) \times \hat{\xi}(\cdot)$ attains the minimum of $J(u(\cdot) \times \xi(\cdot))$.

Reader interested in stochastic optimal control problems can refer to Yong and Zhou [65]. We shall recall the following basic result on BSDE. We begin with the well-known comparison theorem (see Barles, Buckdahn, and Pardoux [7], Proposition 2.6).

Lemma 4 (Comparison theorem). Let $\left(y^{i}, z^{i}\right), i=1,2$, be the solution to the following

$$
y^{i}(t)=\xi^{i}+\int_{t}^{T} f^{i}\left(s, y_{s}^{i}, z_{s}^{i}\right) \mathrm{d} s-\int_{t}^{T} z_{s}^{i} \mathrm{~d} W_{s},
$$

where $\mathbb{E}\left[\left|\xi^{i}\right|^{2}\right]<\infty, f^{i}\left(s, y^{i}, z^{i}\right)$ satisfies the conditions $(\mathrm{A} 2), i=1,2$. Under assumption (A2), BSDE (10) admits a unique adapted solution $\left(y^{i}, z^{i}\right)$, respectively, for $i=1,2$. Furthermore, if

(i) $\xi^{1} \geq \xi^{2}$, a.s.;

(ii) $f^{1}(t, y, z) \geq f^{2}(t, y, z)$, a.e., for any $(t, y, z) \in[0, T] \times \mathbb{R} \times \mathbb{R}^{d}$.

Then, we have: $y_{t}^{1} \geq y_{t}^{2}$, a.s., for all $t \in[0, T]$. 


\section{Dynamic Programming Principle}

In this section, we shall establish the DPP, which plays an important role to derive a new H-J-B inequality. Furthermore, the notion of viscosity solution to dynamic programming inequality will be introduced a moment later. Before this, serval technique lemmas will be presented. From these series of properties of value function (9), we are able to prove it is a viscosity solution.

Definition 5 (Backward Semigroup). For every $s \in\left[t, t_{1}\right]$ with $t \leq t_{1} \leq T$ and $\eta \in L^{2}\left(\Omega, \mathscr{F}_{t_{1}}, P ; \mathbb{R}\right)$, we denote

$$
\mathcal{G}_{s, t_{1}}^{t, x ;, \xi}[\eta]=Y^{t, x ; v, \xi}(s),
$$

where

$$
\left\{\begin{aligned}
\mathrm{d} X_{s}^{t, x ; v, \xi} & =b\left(s, X_{s}^{t, x ; v, \xi}, v_{s}\right) \mathrm{d} s+\sigma\left(s, X_{s}^{t, x ; v, \xi}, v_{s}\right) \mathrm{d} W_{s}+G \mathrm{~d} \xi_{s}, \\
\mathrm{~d} Y_{s}^{t, x ; v, \xi} & =-f\left(t, X_{s}^{t, x ; v, \xi}, Y_{s}^{t, x ; v, \xi}, Z_{s}^{t, x ; v, \xi}, v_{t}\right) \mathrm{d} s+Z_{s}^{t, x ; v, \xi} \mathrm{d} W_{s}-K \mathrm{~d} \xi_{s}, \\
X_{t}^{t, x ; v, \xi} & =x, Y_{t_{1}}^{t, x ; v, \xi}=\eta, \quad 0 \leq t \leq s \leq T .
\end{aligned}\right.
$$

We start from following:

Definition 6. Given any $t \in[0, T]$, a sequence $\left\{A_{i}\right\}_{i=1}^{N} \subset \mathscr{F}_{t}$ is called a partition of $\left(\Omega, \mathscr{F}_{t}\right)$ if $\cup_{i=1}^{N} A_{i}=\Omega$ and $A_{i} \cap A_{j}=\phi$, whenever $i \neq j$.

Lemma 7. Under assumptions (A1)-(A3), the value function $u(t, x)$ defined in (9) is a deterministic function.

Proof. We adopt the idea from Peng [57]. In fact, there exists $\left(v^{l}, \xi^{l}\right)_{l \geq 1}$ such that $u(t, x)=\inf _{l \geq 1} J\left(t, x, v^{l}, \xi^{l}\right)$, a.s. For any $\left((v, \xi),\left(v^{\prime}, \xi^{\prime}\right)\right) \in \mathcal{U}$, we define

$$
\left((v, \xi) \wedge\left(v^{\prime}, \xi^{\prime}\right)\right)_{s}=\left\{\begin{array}{l}
0, s \in[0, t] ; \\
(v, \xi)_{s}, s \in(t, T] \text { on }\left\{J(t, x, v, \xi) \leq J\left(t, x, v^{\prime}, \xi^{\prime}\right)\right\} \\
\left(v^{\prime}, \xi^{\prime}\right)_{s}, s \in(t, T] \text { on }\left\{J(t, x, v, \xi) \geq J\left(t, x, v^{\prime}, \xi^{\prime}\right)\right\}
\end{array}\right.
$$

Hence, $\left((v, \xi) \vee\left(v^{\prime}, \xi^{\prime}\right)\right) \in \mathcal{U}$, moreover, it follows that

$$
J\left(t, x,(v, \xi) \wedge\left(v^{\prime}, \xi^{\prime}\right)\right) \leq J(t, x, v, \xi) \wedge J\left(t, x, v^{\prime}, \xi^{\prime}\right) .
$$

Define $\left(\bar{v}^{1}, \bar{\xi}^{1}\right):=\left(v^{1}, \xi^{1}\right),\left(\bar{v}^{l}, \bar{\xi}^{l}\right):=\left(\bar{v}^{l-1}, \bar{\xi}^{l-1}\right) \wedge\left(v^{l}, \xi^{l}\right)$. Then, we have

$$
u(t, x)=\lim _{l \uparrow \infty} \downarrow J\left(t, x, \bar{v}^{l}, \bar{\xi}^{l}\right) .
$$


Suppose that

$$
\mathbb{E}\left[\left|u(t, x)-J\left(t, x, \bar{v}^{l}, \bar{\xi}^{l}\right)\right|^{2}\right] \leq \frac{1}{l}, l \geq 1
$$

For all $l \geq 1$, there exists $(\tilde{v}, \tilde{\xi})$ where

$$
\begin{aligned}
& \tilde{v}^{l}=\sum_{r, i, j}^{N_{l}-1} \bar{v}_{i, j, r} \mathbf{1}_{A_{j}^{l} \cap B_{i r}^{l}} \mathbf{1}_{\left(t_{i}^{l}, t_{i+1}^{l}\right]}, t=t_{0}^{l} \leq \cdots t_{N_{l}}^{l}=T, \bar{v}_{i, j, r} \in U, \\
& \tilde{\xi}^{l}=\sum_{r, i, j}^{N_{l}-1} \bar{\xi}_{i, j, r} \mathbf{1}_{A_{j}^{l} \cap B_{i r}^{l}} \mathbf{1}_{\left(t_{i}^{l}, t_{i+1}^{l}\right]}, t=t_{0}^{l} \leq \cdots t_{N_{l}}^{l}=T, \bar{\xi}_{i, j, r} \in([0, \infty))^{m},
\end{aligned}
$$

where $\left\{A_{j}^{l}\right\}_{0 \leq j \leq N_{l}-1}$ is a partition of $\left(\Omega, \mathscr{F}_{t}\right),\left\{B_{i r}^{l}\right\}_{0 \leq r \leq N_{l}-1}$ is a partition of $\left(\Omega, \mathscr{F}_{t_{i}^{l}}^{t}\right)$, such that

$$
\mathbb{E}\left[\int_{t}^{T}\left(\left|\bar{v}_{s}^{l}-\tilde{v}_{s}^{l}\right|^{2}+\left|\bar{\xi}_{s}^{l}-\tilde{\xi}_{s}^{l}\right|^{2}\right) \mathrm{d} s++\left|\bar{\xi}_{T}^{l}-\tilde{\xi}_{T}^{l}\right|^{2}\right] \leq \frac{C}{l}, l \geq 1 .
$$

From (A.7), it derives that

$$
\begin{aligned}
& \mathbb{E}\left[\left|J\left(t, x, \tilde{v}^{l}, \tilde{\xi}^{l}\right)-J\left(t, x, \bar{v}^{l}, \bar{\xi}^{l}\right)\right|^{2}\right] \\
\leq & C \mathbb{E}\left[\int_{t}^{T}\left(\left|\bar{v}_{s}^{l}-\tilde{v}_{s}^{l}\right|^{2}+\left|\bar{\xi}_{s}^{l}-\tilde{\xi}_{s}^{l}\right|^{2}\right) \mathrm{d} s+\left|\bar{\xi}_{T}^{l}-\tilde{\xi}_{T}^{l}\right|^{2}\right] \\
\leq & \frac{C}{l}, l \geq 1 .
\end{aligned}
$$

From (11), we get

$$
\mathbb{E}\left[\left|u(t, x)-J\left(t, x, \bar{v}^{l}, \bar{\xi}^{l}\right)\right|^{2}\right] \leq \frac{C}{l}
$$

Now we put

$$
\begin{aligned}
& \tilde{v}^{l, j}=\sum_{r, i, j}^{N_{l}-1} \bar{v}_{i, j, r} \mathbf{1}_{A_{j}^{l} \cap B_{i r}^{l}} \mathbf{1}_{\left(t_{i}^{l}, t_{i+1}^{l}\right]}, t=t_{0}^{l} \leq \cdots t_{N_{l}}^{l}=T, \bar{v}_{i, j, r} \in U, \\
& \tilde{\xi}^{l, j}=\sum_{r, i, j}^{N_{l}-1} \bar{\xi}_{i, j, r} \mathbf{1}_{A_{j}^{l} \cap B_{i r}^{l}} \mathbf{1}_{\left(t_{i}^{l}, t_{i+1}^{l}\right]}, t=t_{0}^{l} \leq \cdots t_{N_{l}}^{l}=T, \bar{\xi}_{i, j, r} \in([0, \infty))^{m} .
\end{aligned}
$$


They are clearly $\mathscr{F}_{s}^{t}$-adapted $U \times([0, \infty))^{m}$ processes. Now consider the following

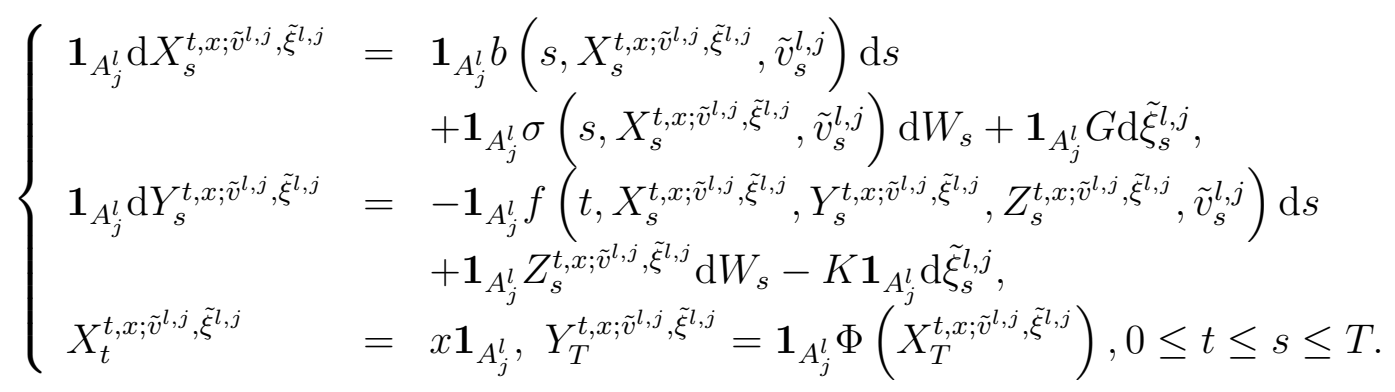

It is easy to derive that

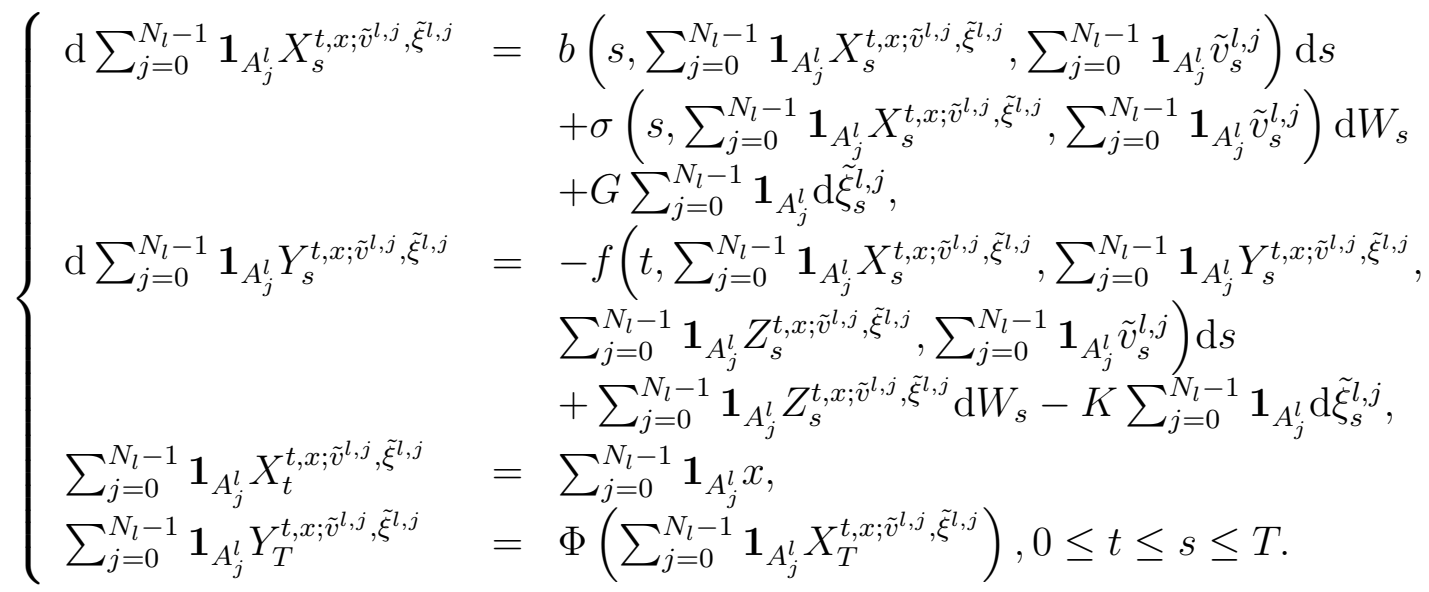

Combining the uniqueness of FBSDEs, we claim that

$$
\begin{aligned}
X_{s}^{t, x ; \tilde{v}^{l}, \tilde{\xi}^{l}} & =\sum_{j=0}^{N_{l}-1} \mathbf{1}_{A_{j}^{l}} X_{s}^{t, x ; \tilde{v}^{l, j}, \tilde{\xi}^{l, j}}, \\
Y_{s}^{t, x ; \tilde{v}^{l}, \tilde{\xi}^{l}} & =\sum_{j=0}^{N_{l}-1} \mathbf{1}_{A_{j}^{l}} Y_{s}^{t, x ; \tilde{v}^{l, j}, \tilde{\xi}^{l, j},}, \\
Z_{s}^{t, x ; \tilde{v}^{l}, \tilde{\xi}^{l}} & =\sum_{j=0}^{N_{l}-1} \mathbf{1}_{A_{j}^{l}} Z_{s}^{t, x ; \tilde{v}^{l, j}, \tilde{\xi}^{l, j}}, s \in[t, T] .
\end{aligned}
$$


From $J\left(t, x ; \tilde{v}^{l, j}, \tilde{\xi}^{l, j}\right):=\left.Y_{s}^{t, x ; \tilde{v}^{l, j}, \tilde{\xi}^{l, j}}\right|_{s=t}$, we conclude that

$$
\begin{aligned}
J\left(t, x ; \tilde{v}^{l}, \tilde{\xi}^{l}\right) & =\left.\sum_{j=0}^{N_{l}-1} \mathbf{1}_{A_{j}^{l}} Y_{s}^{t, x ; \tilde{v}^{l, j}, \tilde{\xi}^{l, j}}\right|_{s=t} \\
& =\sum_{j=0}^{N_{l}-1} \mathbf{1}_{A_{j}^{l}} J\left(t, x ; \tilde{v}^{l, j}, \tilde{\xi}^{l, j}\right) .
\end{aligned}
$$

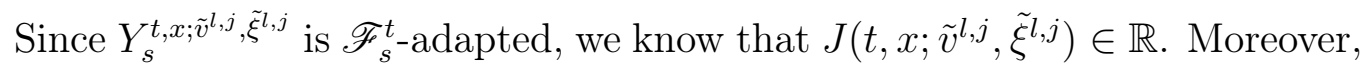

$$
u(t, x) \leq \min _{0 \leq j \leq N_{l-1}} J\left(t, x ; \tilde{v}^{l, j}, \tilde{\xi}^{l, j}\right) \leq J\left(t, x ; \tilde{v}^{l}, \tilde{\xi}^{l}\right) .
$$

Assume that $j=j_{l}$ such that

$$
\min _{0 \leq j \leq N_{l-1}} J\left(t, x ; \tilde{v}^{l, j}, \tilde{\xi}^{l, j}\right)=J\left(t, x ; \tilde{v}^{l, j_{l}}, \tilde{\xi}^{l, j_{l}}\right) .
$$

By (12), we can get

$$
u(t, x)=\lim _{l \rightarrow+\infty} J\left(t, x ; \tilde{v}^{l, j_{l}}, \tilde{\xi}^{l, j_{l}}\right) \in \mathbb{R} .
$$

The proof is completed.

Lemma 8. For any $\varepsilon>0$, there exists a control $(v, \xi) \in \mathcal{U}$, such that

$$
u(t, x) \geq Y_{s}^{t, x ; v, \xi}-\varepsilon,
$$

where $u(t, x)$ is defined in (9)).

Proof. From (12), we can find out an $\mathscr{F}_{s}^{t}$ adapted control pair $\left(v^{i}, \xi^{i}\right)$ such that $u(t, x) \geq Y_{s}^{t, x ; v^{i}, \xi^{i}}-\varepsilon$, a.s. . We denote that $(v, \xi)=\sum_{i=1}^{N}\left(v^{i}, \xi^{i}\right) \mathbf{1}_{A_{i}}$, where $A_{i}$ is a partition of $\left(\Omega, \mathscr{F}_{t}\right)$. Next, we proceed that

$$
\begin{aligned}
Y_{s}^{t, x ; v, \xi} & =\sum_{i=1}^{N} Y_{s}^{t, x ; v^{i}, \xi^{i}} \mathbf{1}_{A_{i}} \\
& \leq \sum_{i=1}^{N}(u(t, x)+\varepsilon) \mathbf{1}_{A_{i}} \\
& =u(t, x)+\varepsilon,
\end{aligned}
$$

from which we get the desired result. 
Lemma 9. Under assumptions (A1)-(A3), the value function $u(t, x)$ defined in (9) obeys the DPP: For any $0 \leq t \leq t+\delta \leq T, x \in \mathbb{R}^{n}$,

$$
u(t, x)=e s s \inf _{(v, \xi) \in \mathcal{U}} \mathcal{G}_{t, t+\delta}^{t, x, v, \xi}\left[u\left(t+\delta, X_{t+\delta}^{t, x, v, \xi}\right)\right] .
$$

Proof. First we have

$$
\begin{aligned}
u(t, x) & =e s s \inf _{(v, \xi) \in \mathcal{U}} \mathcal{G}_{t, T}^{t, x, v, \xi}\left[\Phi\left(X_{T}^{t, x, v, \xi}\right)\right] \\
& =e s s \inf _{(v, \xi) \in \mathcal{U}} \mathcal{G}_{t, t+\delta}^{t, x, v, \xi}\left[Y_{t+\delta}^{t+\delta, X_{t+\delta}^{t, x, v}, v, \xi}\right] .
\end{aligned}
$$

On the one hand, by comparison theorem (lemma 4), we have

$$
u(t, x) \geq e s s \inf _{(v, \xi) \in \mathcal{U}} \mathcal{G}_{t, T}^{t, x, v, \xi}\left[u\left(t+\delta, X_{t+\delta}^{t, x, v, \xi}\right)\right] .
$$

On the other hand, for any $\varepsilon>0$, there exists an admissible control pair $(\bar{v}, \bar{\xi}) \in \mathcal{U}$ such that

$$
u\left(t+\delta, X_{t+\delta}^{t, x ; \bar{v}, \bar{\xi}}\right) \geq Y_{t+\delta}^{t+\delta, X_{t+\delta}^{t, x ; \bar{v}, \bar{\xi}} ; \bar{v}, \bar{\xi}}-\varepsilon, \text { a.s. }
$$

Then, for any $(v, \xi) \in \mathcal{U}$, we define

$$
(\tilde{v}, \tilde{\xi})_{s}= \begin{cases}(v, \xi)_{s}, & s \in[0, t+\delta] \\ (\bar{v}, \bar{\xi})_{s}, & s \in[t+\delta, T] .\end{cases}
$$

Clearly, $(\tilde{v}, \tilde{\xi}) \in \mathcal{U}$. By the comparison theorem (lemma 4) again, we obtain

$$
\begin{aligned}
u(t, x) & \leq \mathcal{G}_{t, t+\delta}^{t, x ; \tilde{\xi}, \tilde{\xi}}\left[Y_{t+\delta}^{\left.t+\delta, X_{t+\delta}^{t, x ; \tilde{v}, \tilde{\xi}} ; \tilde{v}, \tilde{\xi}\right]}\right. \\
& =\mathcal{G}_{t, t+\delta}^{t, x ; v, \xi}\left[Y_{t+\delta}^{t+\delta, X_{t+\delta}^{t, x ; v, \xi} ; \bar{v}, \bar{\xi}}\right] \\
& \leq \mathcal{G}_{t, t+\delta}^{t, x ; v, \xi}\left[u\left(t+\delta, X_{t+\delta}^{t, x ; v, \xi}\right)+\varepsilon\right] \\
& \leq \mathcal{G}_{t, t+\delta}^{t, x ; v, \xi}\left[u\left(t+\delta, X_{t+\delta}^{t, x ; v, \xi}\right)\right]+C \varepsilon
\end{aligned}
$$

where $C$ is independent of control pair. We get the DPP for $u(t, x)$ since the arbitrary of $\varepsilon$.

Lemma 10. Under assumptions (A1)-(A3), the value function $u(t, x)$ defined in (9) admits

$$
\left|u(t, x)-u\left(t^{\prime}, x^{\prime}\right)\right| \leq C\left(\left|x-x^{\prime}\right|+\left|t-t^{\prime}\right|^{\frac{1}{2}}\right), 0 \leq t, t^{\prime} \leq T, x, x^{\prime} \in \mathbb{R}^{n} .
$$


Proof. For any $(t, x) \in[0, T] \times \mathbb{R}^{n}$ and $\forall \delta>0$, from Lemma 9 and Lemma 8, there exists, for any $\varepsilon>0$, an admissible control pair $\left(v^{\varepsilon}, \xi^{\varepsilon}\right)$ such that

$$
\mathcal{G}_{t, t+\delta}^{t, x ; v^{\varepsilon}, \xi^{\varepsilon}}\left[u\left(t+\delta, X_{t+\delta}^{t, x ; v^{\varepsilon}, \xi^{\varepsilon}}\right)\right]-\varepsilon \leq u(t, x) \leq \mathcal{G}_{t, t+\delta}^{t, x ; v^{\varepsilon}, \xi^{\varepsilon}}\left[u\left(t+\delta, X_{t+\delta}^{t, x ; v^{\varepsilon}, \xi^{\varepsilon}}\right)\right] .
$$

From (13), we focus on

$$
u(t, x)-u(t+\delta, x) \geq \Theta_{\delta}^{1}+\Theta_{\delta}^{1}+\varepsilon
$$

where

$$
\begin{aligned}
\Theta_{\delta}^{1} & =\mathcal{G}_{t, t+\delta}^{t, x ; v^{\varepsilon}, \xi^{\varepsilon}}\left[u\left(t+\delta, X_{t+\delta}^{t, x ; v^{\varepsilon}, \xi^{\varepsilon}}\right)\right]-\mathcal{G}_{t, t+\delta}^{t, x ; v^{\varepsilon}, \xi^{\varepsilon}}[u(t+\delta, x)], \\
\Theta_{\delta}^{2} & =\mathcal{G}_{t, t+\delta}^{t, x ; v^{\varepsilon}, \xi^{\varepsilon}}[u(t+\delta, x)]-u(t+\delta, x) .
\end{aligned}
$$

From Lemma 10, we get

$$
\begin{aligned}
\left|\Theta_{\delta}^{1}\right| & \leq C \mathbb{E}^{\mathscr{F} t}\left[\left|u\left(t+\delta, X_{t+\delta}^{t, x ; v^{\varepsilon}, \xi^{\varepsilon}}\right)-u(t+\delta, x)\right|^{2}\right]^{\frac{1}{2}} \\
& \leq C \mathbb{E}^{\mathscr{F}_{t}}\left[\left|X_{t+\delta}^{t, x ; v^{\varepsilon}, \xi^{\varepsilon}}-x\right|^{2}\right]^{\frac{1}{2}} \\
& \leq C\left(1+|x|^{2}\right) \delta .
\end{aligned}
$$

Hence, we deduce that

$$
\Theta_{\delta}^{1} \geq-C(1+|x|) \delta^{\frac{1}{2}}
$$

since $\sqrt{\left(1+|x|^{2}\right)} \leq 1+|x|, \forall x \in \mathbb{R}^{n}$. Next we deal with $\Theta_{\delta}^{2}$. From the definition of DDP, we know that $\Theta_{\delta}^{2}$ can be rewritten as

$$
\begin{aligned}
\Theta_{\delta}^{2}= & \mathbb{E}^{\mathscr{F}_{t}}\left[u(t+\delta, x)+\int_{t}^{t+\delta} f\left(s, X_{s}^{t, x ; v^{\varepsilon}, \xi^{\varepsilon}}, Y_{s}^{t, x ; v^{\varepsilon}, \xi^{\varepsilon}}, Z_{s}^{t, x ; v^{\varepsilon}, \xi^{\varepsilon}}, v_{t}^{\varepsilon}\right) \mathrm{d} s\right. \\
& \left.-\int_{t}^{t+\delta} Z_{s}^{t, x ; v^{\varepsilon}, \xi^{\varepsilon}} \mathrm{d} W_{s}+\int_{t}^{t+\delta} K \mathrm{~d} \xi_{s}^{\varepsilon}\right]-u(t+\delta, x) \\
= & \mathbb{E}^{\mathscr{F}_{t}}\left[\int_{t}^{t+\delta} f\left(s, X_{s}^{t, x ; v^{\varepsilon}, \xi^{\varepsilon}}, Y_{s}^{t, x ; v^{\varepsilon}, \xi^{\varepsilon}}, Z_{s}^{t, x ; v^{\varepsilon}, \xi^{\varepsilon}}, v_{t}^{\varepsilon}\right) \mathrm{d} s+\int_{t}^{t+\delta} K \mathrm{~d} \xi_{s}^{\varepsilon}\right] .
\end{aligned}
$$


Employing Schwartz inequality, we have, for certain constant $C$ not depending on $t$ and $\delta$,

$$
\begin{aligned}
\left|\Theta_{\delta}^{2}\right| \leq & C \delta^{\frac{1}{2}} \mathbb{E}^{\mathscr{F} t}\left[\int_{t}^{t+\delta}\left|f\left(s, X_{s}^{t, x ; v^{\varepsilon}, \xi^{\varepsilon}}, 0,0, v_{t}^{\varepsilon}\right)+\right| Y_{s}^{t, x ; v^{\varepsilon}, \xi^{\varepsilon}} \mid\right. \\
& \left.+\left.\left|Z_{s}^{t, x ; v^{\varepsilon}, \xi^{\varepsilon}}\right||\mathrm{d} s+| \xi_{T}^{\varepsilon}\right|^{2}\right] \\
\leq & C \delta^{\frac{1}{2}} \mathbb{E}^{\mathscr{F} t}\left[\int_{t}^{t+\delta}\left(1+\left|X_{s}^{t, x ; v^{\varepsilon}, \xi^{\varepsilon}}\right|^{2}+\left|Y_{s}^{t, x ; v^{\varepsilon}, \xi^{\varepsilon}}\right|^{2}+\left|Z_{s}^{t, x ; v^{\varepsilon}, \xi^{\varepsilon}}\right|^{2}\right) \mathrm{d} s\right. \\
& \left.+\left|\xi_{T}^{\varepsilon}\right|^{2}\right] \\
\leq & C\left(1+|x|^{2}\right) \delta^{\frac{1}{2}},
\end{aligned}
$$

that is

$$
\Theta_{\delta}^{2} \geq-C\left(1+|x|^{2}\right) \delta^{\frac{1}{2}}
$$

Therefore, combing (14) and (15), we have

$$
u(t, x)-u(t+\delta, x) \geq-C\left(1+|x|^{2}\right) \delta^{\frac{1}{2}}-\varepsilon .
$$

Note that the arbitrary of $\varepsilon>0$, we immitigably get

$$
u(t, x)-u(t+\delta, x) \geq-C\left(1+|x|^{2}\right) \delta^{\frac{1}{2}} .
$$

Repeating the above approach, we can get the desired result basing on the second inequality of (13).

We now study the original optimal control problem via DPP (Lemma 9). Postulate that $u(t, x) \in C^{1,2}\left([0, T] \times \mathbb{R}^{n} ; \mathbb{R}\right)$. Indeed, this hypothesis is really strong, but the main destination here is to derive the so called H-J-B inequality.

Define

$$
\begin{aligned}
\mathcal{L}(t, x, v) \Psi & =\frac{1}{2} \operatorname{Tr}\left(\sigma \sigma^{*}(t, x, v) D^{2} \Psi\right)+\langle D \Psi, b(t, x, v)\rangle \\
(t, x, v) & \in[0, T] \times \mathbb{R}^{n} \times U, \Psi \in C^{1,2}\left([0, T] \times \mathbb{R}^{n}\right) .
\end{aligned}
$$


For any $\Psi \in C^{1,2}\left([0, T] \times \mathbb{R}^{n} ; \mathbb{R}\right)$, by virtue of Doléans-Dade-Meyer formula (see [36, 17, 63]), we have

$$
\begin{aligned}
\Psi\left(s, X_{s}\right)= & \Psi(t, x)+\int_{t}^{s} \Psi_{t}\left(r, X_{r}\right)+\mathcal{L}\left(r, X_{r}, v\right) \Psi\left(r, X_{r}\right) \mathrm{d} r \\
& +\int_{t}^{s} \Psi_{x}\left(r, X_{r}\right) \sigma\left(r, X_{r}, v_{r}\right) \mathrm{d} W_{r}+\int_{t}^{s} \Psi_{x}\left(r, X_{r}\right) G \mathrm{~d} \xi_{r} \\
& +\sum_{t \leq r \leq s}\left\{\Psi\left(r, X_{r+}\right)-\Psi\left(r, X_{r}\right)-\Psi_{x}\left(r, X_{r}\right) \Delta X_{r}\right\}
\end{aligned}
$$

Taking expectation, we get

$$
\begin{aligned}
& \mathbb{E}\left[\Psi\left(s, X_{s}\right)\right] \\
= & \Psi\left(t, X_{t}\right)+\mathbb{E}\left[\int_{t}^{s} \Psi_{x}\left(r, X_{r}\right) G \mathrm{~d} \xi_{r}\right] \\
+ & \mathbb{E}\left[\int_{t}^{s} \Psi_{t}\left(r, X_{r}\right)+\mathcal{L}(t, x, v) \Psi\left(r, X_{r}\right) \mathrm{d} r\right] \\
+ & \mathbb{E}\left[\sum_{t \leq r \leq s}\left\{\Psi\left(r, X_{r+}\right)-\Psi\left(r, X_{r}\right)-\Psi_{x}\left(r, X_{r}\right) \Delta X_{r}\right\}\right] .
\end{aligned}
$$

Now according to the DPP (Lemma 9), it follows

$$
\begin{aligned}
u(t, x)= & \inf _{v(\cdot) \times \xi(\cdot) \in \mathcal{U}} \mathbb{E}\left[\int_{t}^{s} f\left(r, X_{r}^{t, x ; v, \xi}, Y_{r}^{t, x ; v, \xi}, Z_{r}^{t, x ; v, \xi}, v_{r}\right) \mathrm{d} r\right. \\
& \left.-\int_{t}^{s} Z_{r}^{t, x ; v, \xi} \mathrm{d} W_{r}+\int_{t}^{s} K \mathrm{~d} \xi_{r}+u\left(s, X_{s}^{t, x ; v, \xi}\right)\right] .
\end{aligned}
$$

Hence, by Itô's formula (17) again, (18) yields

$$
\begin{aligned}
0= & \inf _{v(\cdot) \times \xi(\cdot) \in \mathcal{U}} \mathbb{E}\left[\int _ { t } ^ { s } \left[u_{t}\left(r, X_{r}^{t, x ; v, \xi}\right)+\mathcal{L}\left(r, X_{r}^{t, x ; v, \xi}, v_{r}\right) u\left(r, X_{r}^{t, x ; v, \xi}\right)\right.\right. \\
& \left.+f\left(r, X_{r}^{t, x ; v, \xi}, Y_{r}^{t, x ; v, \xi}, Z_{r}^{t, x ; v, \xi}, v_{r}\right)\right] \mathrm{d} r \\
& +\int_{t}^{s}\left(u_{x}^{\top}\left(r, X_{r}^{t, x ; v, \xi}\right) G+K\right) \mathrm{d} \xi_{r} \\
& +\int_{t}^{s}\left(u_{x}^{\top}\left(r, X_{r}\right) \sigma\left(r, X_{r}, v_{r}\right)-Z_{r}^{t, x ; v, \xi}\right) \mathrm{d} W_{r}
\end{aligned}
$$




$$
\begin{aligned}
& +\sum_{t \leq r \leq s}\left[u\left(r, X_{r+}^{t, x ; v, \xi}\right)-u\left(r, X_{r}^{t, x ; v, \xi}\right)\right. \\
& \left.\left.-u_{x}\left(r, X_{r}^{t, x ; v, \xi}\right) \Delta X_{r}^{t, x ; v, \xi}\right]\right] .
\end{aligned}
$$

From (19), it is possible to give the following definition of classical solution.

Definition 11 (Classical Solution). Consider a function $V(t, x) \in C^{1,2}\left([0, T] \times \mathbb{R}^{n} ; \mathbb{R}\right)$ and define that

$$
\mathcal{D}_{t}(V):=\left\{x \in \mathbb{R}^{n}: V_{x}^{\top}(t, x) G_{t}+K>0\right\} .
$$

We can say $V$ is a classical solution of the dynamic programming inequality (19), if for every $(t, x) \in[0, T] \times \mathcal{D}_{t}(V), V$ admits

$\frac{\partial}{\partial t} V(t, x)+\min _{v \in U}\{\mathcal{L}(t, x, v) V(t, x)+f(t, x, V(t, x), \nabla V(t, x) \sigma(t, x, v), v)\}=0$,

and

$$
V(T, x)=\Phi(x), x \in \mathbb{R}^{n} .
$$

Moreover, for any $(t, x, v) \in[0, T] \times \mathbb{R}^{n} \times U$,

$$
V_{x}^{\top}(t, x) G+K \geq 0
$$

and

$$
\frac{\partial}{\partial t} V(t, x)+\mathcal{L}(t, x, v) V(t, x)+f(t, x, V(t, x), \nabla V(t, x) \sigma(t, x, v), v) \geq 0 .
$$

Remark 12. In the Definition (11), $V_{x}^{\top}(t, x) G+K \geq 0$ means $\left(V_{x}^{\top}(t, x) G\right)^{i}+$ $K^{i} \geq 0, i=1,2, \ldots, m$, where $(\cdot)^{i}$ denotes the ith coordinate of the point in $\mathbb{R}^{m}$. Now we introduce the following:

$$
\begin{aligned}
& 0=\min \left\{\frac{\partial}{\partial t} V(t, x)\right. \\
& +\min _{v \in U}\{\mathcal{L}(t, x, v) V(t, x)+f(t, x, V(t, x), \nabla V(t, x) \sigma(t, x, v), v)\} \\
& \left.V_{x}^{\top}(t, x) G+K\right\} .
\end{aligned}
$$


We shall prove that, under certain assumptions, the classical solution $V$ to H-J-B inequality (21) is just the optimal value function with optimal control pair.

Theorem 13 (Verification Theorem). Suppose that $V$ is a classical solution of the $H-J-B$ inequality (21) such that for some $l>1,|V(t, x)| \leq$ $C\left(1+|x|^{l}\right)$. Then for any $[0, T] \times \mathbb{R}^{n},(v, \xi) \in \mathcal{U}$ :

$$
V(t, x) \leq J(t, x, v, \xi) .
$$

Furthermore, if there exists $(\hat{v}, \hat{\xi}) \in \mathcal{U}$ such that

$$
\begin{gathered}
P\left\{\left(r, X_{r}^{t, x ; \hat{v}, \hat{\xi}}\right) \in \mathcal{D}_{r}(V), 0 \leq r \leq T\right\}=1, \\
P\left\{\int_{[t, T]}\left[V_{x}^{\top}(r, x) G+K\right] \mathrm{d} r=0\right\}=1, \\
1=P\left\{\left(s, X_{s+}^{t, x ; \hat{v}, \hat{\xi}}\right) \in \mathcal{D}_{r}(V), t \leq s \leq T:\right. \\
\hat{v}_{s} \in \min _{v \in U}\left[V_{t}\left(s, X_{s}^{t, x ; v, \xi}\right)+\mathcal{L}\left(s, X_{s+}^{t, x ; \hat{v}, \hat{\xi}}, v\right) V\left(s, X_{s+}^{t, x ; \hat{v}, \hat{\xi}}\right)\right. \\
+f\left(s, X_{s+}^{t, x ; \hat{v}, \hat{\xi}}, V\left(t, X_{s+}^{t, x ; \hat{v}, \hat{\xi}}\right),\right. \\
\left.\left.\left.\nabla V\left(s, X_{s+}^{t, x ; \hat{v}, \hat{\xi}}\right) \sigma\left(s, X_{s+}^{t, x ; \hat{v}, \hat{\xi}}, v\right), v\right)\right]\right\}
\end{gathered}
$$

and

$$
P\left\{V\left(s, X_{s}^{t, x ; \hat{v}, \hat{\xi}}\right)=V\left(s, X_{s+}^{t, x ;} \hat{v}, \hat{\xi}\right)+K \Delta \hat{\xi}_{s}, t \leq s \leq T\right\}=1 .
$$

Then

$$
V(t, x)=J(t, x ; \hat{v}(\cdot), \hat{\xi}(\cdot)) .
$$


Proof. For any $(t, x) \in[0, T] \times \mathbb{R}^{n}$ and $(v, \xi) \in \mathcal{U}$, applying Doléans-DadeMeyer formula to $V$, we have

$$
\begin{aligned}
V(t, x)= & \mathbb{E}\left[\Phi\left(X_{T}^{t, x ; v, \xi}\right)\right]-\mathbb{E}\left[\int_{t}^{s}\left(r, X_{r}^{t, x ; v, \xi}\right)\right. \\
& \left.+\mathcal{L}\left(r, X_{r}^{t, x ; v, \xi}, v\right) V\left(r, X_{r}^{t, x ; v, \xi}\right) \mathrm{d} r\right] \\
& -\mathbb{E}\left[\int_{t}^{s} V_{x}\left(r, X_{r}^{t, x ; v, \xi}\right) G \mathrm{~d} \xi_{r}\right] \\
& -\mathbb{E}\left[\sum _ { t \leq r \leq s } \left[V\left(r, X_{r+}^{t, x ; v, \xi}\right)-V\left(r, X_{r}^{t, x ; v, \xi}\right)\right.\right. \\
& \left.\left.-V_{x}\left(r, X_{r}^{t, x ; v, \xi}\right) \Delta X_{r}^{t, x ; v, \xi}\right]\right]
\end{aligned}
$$

Note that $\Delta X_{r}^{t, x ; v, \xi}=G \Delta \xi_{r}$, and $X_{r+}^{t, x ; v, \xi}=X_{r}^{t, x ; v, \xi}+\Delta X_{r}^{t, x ; v, \xi}=X_{r}^{t, x ; v, \xi}+$ $G \Delta \xi_{r}$. Thus

$$
\begin{aligned}
& -\mathbb{E}\left[\int_{t}^{s} V_{x}\left(r, X_{r}^{t, x ; v, \xi}\right) G \mathrm{~d} \xi_{r}\right]+\mathbb{E}\left[V_{x}\left(r, X_{r}^{t, x ; v, \xi}\right) \Delta X_{r}^{t, x ; v, \xi}\right] \\
= & -\mathbb{E}\left[\int_{t}^{s} V_{x}\left(r, X_{r}^{t, x ; v, \xi}\right) G \mathrm{~d} \xi_{r}^{c}\right] \\
\leq & \mathbb{E}\left[\int_{t}^{s} K \mathrm{~d} \xi_{r}^{c}\right] .
\end{aligned}
$$

We now deal the term

$$
\begin{aligned}
& -\mathbb{E}\left[\sum_{t \leq r \leq s}\left\{V\left(r, X_{r+}^{t, x ; v, \xi}\right)-V\left(r, X_{r}^{t, x ; v, \xi}\right)\right\}\right] \\
= & -\mathbb{E}\left[\sum_{t \leq r \leq s}\left\{\int_{0}^{1} V_{x}\left(r, X_{r}^{t, x ; v, \xi}+\theta \Delta X_{r}^{t, x ; v, \xi}\right) G \Delta \xi_{r} \mathrm{~d} \theta\right\}\right] \\
\leq & \mathbb{E}\left[K \Delta \xi_{r}\right] .
\end{aligned}
$$


Combining (28) and (29), we have

$$
\begin{aligned}
V(t, x) \leq & \mathbb{E}\left[\Phi\left(X_{T}^{t, x ; v, \xi}\right)+\int_{t}^{s} f\left(r, X_{r}^{t, x ; v, \xi}, Y_{r}^{t, x ; v, \xi}, Z_{r}^{t, x ; v, \xi}, v_{r}\right) \mathrm{d} r\right. \\
& \left.+\int_{t}^{s} K \mathrm{~d} \xi_{r}\right]
\end{aligned}
$$

Now we take $(\hat{v}, \hat{\xi}) \in \mathcal{U}$ such that $(22)-(25)$ hold. Then repeating above method, we have

$$
\begin{aligned}
V(t, x) \leq & \mathbb{E}\left[\Phi\left(X_{T}^{t, x ; \hat{v}, \hat{\xi}}\right)+\int_{t}^{s} f\left(r, X_{r}^{t, x ; \hat{v}, \hat{\xi}}, Y_{r}^{t, x ; \hat{v}, \hat{\xi}}, Z_{r}^{t, x ; \hat{v}, \hat{\xi}}, \hat{v}_{r}\right) \mathrm{d} r\right. \\
& \left.+\int_{t}^{s} K \mathrm{~d} \hat{\xi}_{r}\right] .
\end{aligned}
$$

We thus complete the proof.

Remark 14. As observed in Theorem 13, it provides a possible way to check a pair of potential singular controls is optimal or not. Anyhow, note that Section 6 in [63] is devoted to this issue via constructing an optimal control. In this sense, our verification theorem seems to be rather weak, since we do not know whether $(\hat{v}, \hat{\xi})$ exists or not. Regarding to this topic, we shall discus in Remark 22 below. Verification theorem under non-smooth situation, namely, viscosity approach can be found in Zhang [6\%].

In the classical optimal control theory of stochastic systems, the value function is a solution to the corresponding H-J-B equation whenever it has sufficient regularity (Fleming and Rishel [32], Krylov [46]). Nevertheless when it is only known that the value function is continuous, then, the value function is a solution to the H-J-B equation in the viscosity sense (see Lions [20]). From now on, we will relate the value function of above recursive optimal singular control problem with the following nonlinear second-order parabolic PDEs with "gradient constraints":

$$
\left\{\begin{array}{l}
\min \left(u_{x}^{\top}(t, x) G+K, \frac{\partial}{\partial t} u(t, x)\right. \\
\left.+\min _{v \in U} \mathcal{L}(t, x, v) u(t, x)+f(t, x, u(t, x), \nabla u(t, x) \sigma(t, x, v), v)\right)=0, \\
u(T, x)=\Phi(x), 0 \leq t \leq T .
\end{array}\right.
$$


Before our main result, we state the following interesting result whenever the value function defined by (9) is sufficiently smooth.

Lemma 15. Assume that $u(t, x) \in C^{1,2}\left([0, T] \times \mathbb{R}^{n} ; \mathbb{R}\right)$ is a classical solution to $H$ - $J-B$ inequality (30). For any $(t, x) \in \mathcal{D}_{t}(u)$, we have

$$
u(t, x) \leq u(t, x+G h)+K h,
$$

for each $h \in \mathbb{R}_{+}^{m}$. Furthermore, if the equality holds for some $h \in \mathbb{R}_{+}^{m}$, then there exists $\bar{h}=(\bar{h})^{i} \in \mathbb{R}_{+}^{m}$ such that $(\bar{h})^{i} \leq(h)^{i}, 1 \leq i \leq m$ and the equality also holds.

Proof. From (20), it is easy to show

$$
\begin{aligned}
u(t, x+G h)-u(t, x) & =\int_{0}^{1} u_{x}(t, x+\theta G h) G h \mathrm{~d} \theta \\
& \geq-K h .
\end{aligned}
$$

We now proceed the second result. If the equality holds as an inequality for some $h \in \mathbb{R}_{+}^{m}$, then we take $\bar{h}$ such that $\bar{h}^{i} \leq h^{i}, 1 \leq i \leq m$. It follows that

$$
\begin{aligned}
u(t, x)-K h & =u(t, x+G h) \\
& \geq u(t, x+G \bar{h})-K(h-\bar{h}) \\
& \geq u(t, x)-K \bar{h}-K(h-\bar{h}) \\
& =u(t, x)-K h .
\end{aligned}
$$

Thus

$$
u(t, x)=u(t, x+G \bar{h})+K \bar{h} .
$$

The proof is completed.

We now focus on the value function $u$ defined in (9), satisfying continuity (see Lemma 10). Inspired by the inequality (31), we may define a freedom domain for H-J-B inequality (30). The following lemma will indicate that, for H-J-B inequality (30), there exists a region, called inaction region, such that if the optimal state process starts from outside this region, then apply the singular control to bring it to the region immediately, and then keep it inside this region from then on. In this interiors of the region, the optimal singular control should not produce any jump. 
Lemma 16. Define

$$
\tilde{\mathcal{D}}_{t}(u):=\left\{x \in \mathbb{R}^{n}: u(t, x)<u(t, x+G h)+K h, h \in \mathbb{R}_{+}^{m}, h \neq 0\right\} .
$$

Then the optimal state process $X^{t, x ; \hat{v}, \hat{\xi}}$ is continuous whenever $\left(r, X_{r}^{t, x ; \hat{v}, \hat{\xi}}\right) \in$ $\tilde{\mathcal{D}}_{r}(u)$. To be precise, we have

$$
P\left(\Delta X_{r}^{t, x ; \hat{v}, \hat{\xi}} \neq 0, \quad X_{r}^{t, x ; \hat{v}, \hat{\xi}} \in \tilde{\mathcal{D}}_{r}(u)\right)=0, t \leq r \leq T .
$$

Proof. For any $r>t$, we first have $\Delta X_{r}^{t, x ; v, \xi}=G \cdot \Delta \xi_{r}$, which yields that $X_{r+}^{t, x ; v, \xi}=X_{r}^{t, x ; v, \xi}+G \cdot \Delta \xi_{r}$. On the other hand, Lemma [10] implies that

$$
u\left(r, X_{r+}^{t, x ; \hat{v}, \hat{\xi}}\right)=\lim _{r^{\prime} \downarrow \downarrow r} u\left(r^{\prime}, X_{r^{\prime}}^{t, x ; \hat{v}, \hat{\xi}}\right) .
$$

By DPP (Lemma 9), we have, for $r^{\prime}>r$,

$$
\begin{aligned}
u(t, x)= & \mathbb{E}^{\mathscr{F} t}\left[\int_{t}^{r^{\prime}} f\left(s, X_{s}^{t, x ; \hat{v}, \hat{\xi}}, Y_{s}^{t, x ; \hat{v}, \hat{\xi}}, Z_{s}^{t, x ; \hat{v}, \hat{\xi}}, \hat{v}_{s}\right) \mathrm{d} s\right. \\
& \left.+\int_{t}^{r^{\prime}} K d \hat{\xi}_{s}+u\left(r^{\prime}, X_{r^{\prime}}^{t, x ; \hat{v}, \hat{\xi}}\right)\right] .
\end{aligned}
$$

Letting $r^{\prime} \rightarrow r$, we immediately have

$$
\begin{aligned}
& \int_{t}^{r^{\prime}} f\left(s, X_{s}^{t, x ; \hat{v}, \hat{\xi}}, Y_{s}^{t, x ; \hat{v}, \hat{\xi}}, Z_{s}^{t, x ; \hat{v}, \hat{\xi}}, \hat{v}_{s}\right) \mathrm{d} s \\
\rightarrow & \int_{t}^{r} f\left(s, X_{s}^{t, x ; \hat{v}, \hat{\xi}}, Y_{s}^{t, x ; \hat{v}, \hat{\xi}}, Z_{s}^{t, x ; \hat{v}, \hat{\xi}}, \hat{v}_{s}\right) \mathrm{d} s, \\
& \int_{t}^{r^{\prime}} K d \hat{\xi}_{s} \rightarrow \int_{t}^{r} K d \hat{\xi}_{s} .
\end{aligned}
$$

We proceed our proof by contradiction. Suppose that

$$
P\left(\Delta X_{r}^{t, x ; v, \xi} \neq 0, \quad\left(r, X_{r}^{t, x ; \hat{v}, \hat{\xi}}\right) \in \tilde{\mathcal{D}}_{r}(u)\right)>0, t \leq r \leq T .
$$


Then, from Lemma 10 and (32), we have

$$
\begin{aligned}
u(t, x)= & \mathbb{E}^{\mathscr{F} t}\left[\int_{t}^{r} f\left(s, X_{s}^{t, x ; \hat{v}, \hat{\xi}}, Y_{s}^{t, x ; \hat{v}, \hat{\xi}}, Z_{s}^{t, x ; \hat{v}, \hat{\xi}}, \hat{v}_{s}\right) \mathrm{d} s+\int_{t}^{r} K \mathrm{~d} \hat{\xi}_{s}\right. \\
& \left.+K \cdot \Delta \hat{\xi}_{r}+u\left(r, X_{r}^{t, x ; \hat{v}, \hat{\xi}}+G \cdot \Delta \hat{\xi}_{r}\right)\right] \\
> & \mathbb{E}^{\mathscr{F}_{t}}\left[\int_{t}^{r} f\left(s, X_{s}^{t, x ; \hat{v}, \hat{\xi}}, Y_{s}^{t, x ; \hat{v}, \hat{\xi}}, Z_{s}^{t, x ; \hat{v}, \hat{\xi}^{\prime}}, \hat{v}_{s}\right) \mathrm{d} s+\int_{t}^{r} K \mathrm{~d} \hat{\xi}_{s}\right. \\
& \left.+u\left(r, X_{r}^{t, x ; \hat{v}, \hat{\xi}}\right)\right],
\end{aligned}
$$

which leads to a contradiction to Lemma 9, Note that the strict inequity holds whenever $\left(r, X_{r}^{t, x ; \hat{v}, \hat{\xi}}\right) \in \tilde{\mathcal{D}}_{r}(u), t \leq r \leq T$, a.s.

Remark 17. We say $\tilde{\mathcal{D}}_{t}(u)$ is the domain of $\xi$-inaction, while its complement the region of $\xi$-action. The optimal process can be described via these two regions. For $\left(t, X_{t}^{t, x ; v, \xi}\right)=(t, x) \notin \tilde{\mathcal{D}}_{t}(u)$, the optimal control processes make $(t, x)$ to jump instantly to the boundary of $\tilde{\mathcal{D}}_{t}(u)$. In fact, by Lemma 15. if $\left(t, X_{t}^{t, x ; v, \xi}\right) \notin \tilde{\mathcal{D}}_{t}(u)$, there exists some $\bar{h}=(\bar{h})^{i} \in \mathbb{R}_{+}^{m}$ such that $(\bar{h})^{i} \leq(h)^{i}, 1 \leq i \leq m$,

$$
u(t, x) \leq u(t, x+G \bar{h})+K \bar{h} .
$$

Hence, if $u(t, x) \in C^{1,2}\left([0, T] \times \mathbb{R}^{n} ; \mathbb{R}\right)$, we have $u_{x}^{\top}(t, x) G+K \geq 0$, which means (20) always holds.

Our aim is to prove that the value function $u(t, x)$ introduced by (9), is the viscosity solution of the H-J-B variational inequality (30) of singular optimal problem. We first recall the definition of a viscosity solution for H-J-B variational inequality (30) from [20].

Definition 18. Let $u(t, x) \in C\left([0, T] \times \mathbb{R}^{n}\right)$ and $(t, x) \in[0, T] \times \mathbb{R}^{n}$. For every $\varphi \in C^{1,2}\left([0, T] \times \mathbb{R}^{n}\right)$

(1) for each local maximum point $\left(t_{0}, x_{0}\right)$ of $u-\varphi$ in the interior of $[0, T] \times \mathbb{R}^{n}$, we have

$$
\min \left(\varphi_{x}^{\top} G+K, \frac{\partial \varphi}{\partial t}+\min _{v \in U}\left\{\mathcal{L} \varphi+f\left(t_{0}, x_{0}, \varphi, \nabla \varphi \sigma, v\right)\right\}\right) \geq 0
$$


at $\left(t_{0}, x_{0}\right)$, i.e., $u$ is a subsolution.

(2) for each local minimum point $\left(t_{0}, x_{0}\right)$ of $u-\varphi$ in the interior of $[0, T] \times \mathbb{R}^{n}$, we have

$$
\min \left(\varphi_{x}^{\top} G+K, \frac{\partial \varphi}{\partial t}+\min _{v \in U}\left\{\mathcal{L} \varphi+f\left(t_{0}, x_{0}, \varphi, \nabla \varphi \sigma, v\right)\right\}\right) \leq 0
$$

at $\left(t_{0}, x_{0}\right)$, i.e., $u$ is a supersolution.

(3) $u(t, x) \in C\left([0, T] \times \mathbb{R}^{n}\right)$ is said to be a viscosity solution of (30) if it is both a viscosity sub and supersolution.

We have the other definition which will be useful to verify the viscosity solutions. Below, $\mathbb{S}^{n}$ will denote the set of $n \times n$ symmetric matrices.

Definition 19. Let $u(t, x) \in C\left([0, T] \times \mathbb{R}^{n}\right)$ and $(t, x) \in[0, T] \times \mathbb{R}^{n}$. We denote by $\mathcal{P}^{2,+} u(t, x)$, the "parabolic superjet" of $u$ at $(t, x)$ the set of triples $(p, q, X) \in \mathbb{R} \times \mathbb{R}^{n} \times \mathbb{S}^{n}$ which are such that

$$
\begin{aligned}
u(s, y) \leq & u(t, x)+p(s-t)+\langle q, x-y\rangle \\
& +\frac{1}{2}\langle X(y-x), y-x\rangle+o\left(|s-t|+|y-x|^{2}\right) .
\end{aligned}
$$

Similarly, we denote by $\mathcal{P}^{2,-} u(t, x)$, the "parabolic subjet" of $u$ at $(t, x)$ the set of triples $(p, q, X) \in \mathbb{R} \times \mathbb{R}^{n} \times \mathbb{S}^{n}$ which are such that

$$
\begin{aligned}
u(s, y) \geq & u(t, x)+p(s-t)+\langle q, x-y\rangle \\
& +\frac{1}{2}\langle X(y-x), y-x\rangle+o\left(|s-t|+|y-x|^{2}\right) .
\end{aligned}
$$

For convenience, specifically dealing with the uniqueness of viscosity solution, we define

$$
\begin{aligned}
\mathcal{H}(t, x, q, X)= & \inf _{v \in U}\left\{\frac{1}{2} \operatorname{Tr}\left(\sigma \sigma^{*}(t, x, v) X\right)+\langle q, b(t, x, v)\rangle\right. \\
& +f(t, x, u(t, x), q \sigma(t, x, v))\} .
\end{aligned}
$$

Note that, when the terms $\left(\frac{\partial \varphi}{\partial t}, \varphi_{x}, \varphi_{x x}\right)$ in Definition 18 are taken replace by $(p, q, X)$, we also have the other equivalent definition of viscosity solution, that is, $\left(\frac{\partial \varphi}{\partial t}, \varphi_{x}, \varphi_{x x}\right)$ with local maximum point $\left(t_{0}, x_{0}\right)$ of $u-\varphi$ (local minimum point $\left(t_{0}, x_{0}\right)$ of $\left.u-\varphi\right)$ is corresponding to $(p, q, X) \in \mathcal{P}^{2,+} u(t, x)$ $\left((p, q, X) \in \mathcal{P}^{2,-} u(t, x)\right)$, respectively. For more details, see Fleming and Soner [31], Lemma 4.1.

We are now in the position to assert following: 
Theorem 20. The value function $u(\cdot, \cdot)$ defined in (9) is a continuous viscosity solution to (30).

Proof. From Lemma [10, we have $u \in C\left([0, T] \times \mathbb{R}^{n}\right)$ and clearly $u(T, x)=$ $\Phi(x)$. We first show $u$ is subsolution. To this end, we suppose that $\varphi \in$ $C^{1,2}\left([0, T] \times \mathbb{R}^{n}\right)$, for any $(t, x) \in[0, T] \times \mathbb{R}^{n}$ such that $u-\varphi$ attains a global maximum at $(t, x)$ since the value function is continuous and linear growth (see Lemma 10). Without loss of generality, we may also suppose that $u(t, x)=\varphi(t, x)$, then

$$
u(\bar{t}, \bar{x})-u(t, x) \leq \varphi(\bar{t}, \bar{x})-\varphi(t, x), \quad(t, x) \neq(\bar{t}, \bar{x}) \in[0, T] \times \mathbb{R}^{n} .
$$

Suppose that (35) does not hold which means one of the follows is valid:

$$
\begin{gathered}
\frac{\partial \varphi}{\partial t}(t, x)+\min _{v \in U} \mathcal{L}(t, x, v) \varphi(t, x)+f(t, x, \varphi(t, x), \nabla \varphi(t, x) \sigma(t, x, v), v)<0, \\
\varphi_{x}^{\top}(t, x) G_{t}+K<0 \text { for some } i, 1 \leq i \leq m .
\end{gathered}
$$

We start to deal with (39) first. If (39) is true for some $i$, then we can take $h^{i}$ small enough such that

$$
\varphi\left(t, x+G^{i} h^{i}\right)-\varphi(t, x)<-K h^{i},
$$

where $G^{i}$ denotes the $i$ th column of $G(n \times m$ matrix). Hence, (37) can imply that

$$
u\left(t, x+G^{i} h^{i}\right)-u(t, x)<-K h^{i} .
$$

If we set $h=\left(0, \ldots, h^{i}, \ldots 0\right)$, then we have $u(t, x)>u(t, x+G h)+K h$, which is obviously contradiction to (31) in Lemma 15. Now we have obtained

$$
\varphi_{x}^{\top}(t, x) G+K \geq 0, \quad(t, x) \in[0, T] \times \mathbb{R}^{n} .
$$

Based on this result, we are able to construct the second contradiction for (38). Thus by virtue of DPP (see Lemma 9), we have

$$
\varphi(t, x)=u(t, x)=e s s \inf _{(v, \xi) \in \mathcal{U}} \mathcal{G}_{t, t+\delta ; v}^{t, \xi}\left[u\left(t+\delta, X_{t+\delta}^{t, x ; v, \xi}\right)\right], 0 \leq \delta \leq T-t .
$$

To abbreviate the notations, we put,

$$
\begin{aligned}
F(s, x, y, z, v)= & \frac{\partial}{\partial s} \varphi(s, x)+\mathcal{L}(t, x, v) \varphi(t, x) \\
& +f(s, x, y+\varphi(s, x), z+\nabla \varphi(s, x) \sigma(s, x, v), v),
\end{aligned}
$$


where $(s, x, y, z, v) \in[0, T] \times \mathbb{R}^{n} \times \mathbb{R} \times \mathbb{R}^{d} \times U$. We now introduce the following BSDE on interval $[t, t+\delta], 0 \leq \delta \leq T-t$,

$Y_{s}^{1, v, \xi}=\int_{s}^{t+\delta} F\left(s, X_{s}^{t, x ; v, \xi}, Y_{s}^{1, v, \xi}, Z_{s}^{1, v, \xi}, v_{s}\right) \mathrm{d} s-\int_{s}^{t+\delta} Z_{s}^{1, v, \xi} \mathrm{d} W_{s}+\int_{s}^{t+\delta} K \mathrm{~d} \xi_{r}$,

where $(v, \xi) \in \mathcal{U}$. Clearly $Y_{t+\delta}^{1, v, \xi}=0$. We check that $F\left(s, X_{s}^{t, x ; v, \xi}, y, z, v_{s}\right)$ satisfies the assumption (A2), thus we claim that Eq. (41) admits a unique adapted solution. In order to characterize the process $Y_{s}^{1, v, \xi}$, we will study a new BSDE as follows:

$$
\begin{aligned}
Y_{s}^{v, \xi}= & \varphi\left(t+\delta, X_{t+\delta}^{t, x ; v, \xi}\right)+\int_{s}^{t+\delta} f\left(r, X_{r}^{t, x ; v, \xi}, Y_{r}^{v, \xi}, Z_{r}^{v, \xi}, v_{r}\right) \mathrm{d} r \\
& -\int_{s}^{t+\delta} Z_{r}^{v, \xi} \mathrm{d} W_{s}+\int_{s}^{t+\delta} K \mathrm{~d} \xi_{r} .
\end{aligned}
$$

Applying Itô's formula to $\varphi(t, x)$ on interval $[t, t+\delta]$, we have

$$
\begin{aligned}
\varphi\left(s, X_{s}^{t, x ; v, \xi}\right)= & \varphi\left(t+\delta, X_{t+\delta}^{t, x ; v, \xi}\right) \\
& -\int_{s}^{t+\delta}\left[\frac{\partial}{\partial s} \varphi(r, x)+\mathcal{L}\left(r, X_{r}^{t, x ; v, \xi}, v_{r}\right) \varphi\left(r, X_{r}^{t, x ; v, \xi}\right)\right] \mathrm{d} r \\
& -\int_{s}^{t+\delta} \nabla \varphi^{\top}\left(r, X_{r}^{t, x ; v, \xi}\right) G \mathrm{~d} \xi_{r} \\
& -\int_{s}^{t+\delta} \nabla \varphi\left(r, X_{r}^{t, x ; v, \xi}\right) \sigma\left(r, X_{r}^{t, x ; v, \xi}, v_{r}\right) \mathrm{d} W_{r} \\
& -\sum_{s \leq r \leq t+\delta}\left[\varphi\left(r, X_{r+}^{t, x ; v, \xi}\right)-\varphi\left(r, X_{r}^{t, x ; v, \xi}\right)\right. \\
& \left.-\nabla \varphi^{\top}\left(r, X_{r}^{t, x ; v, \xi}\right) \Delta X_{r}^{t, x ; v, \xi}\right] .
\end{aligned}
$$

Since

$$
\begin{aligned}
& -\int_{s}^{t+\delta} \nabla \varphi^{\top}\left(r, X_{r}^{t, x ; v, \xi}\right) G \mathrm{~d} \xi_{r}+\sum_{s \leq r \leq t+\delta} \nabla \varphi^{\top}\left(r, X_{r}^{t, x ; v, \xi}\right) \Delta X_{r}^{t, x ; v, \xi} \\
= & -\int_{s}^{t+\delta} \nabla \varphi^{\top}\left(r, X_{r}^{t, x ; v, \xi}\right) G \mathrm{~d} \xi_{r}^{c} \\
\leq & \int_{s}^{t+\delta} K \mathrm{~d} \xi_{r}^{c}
\end{aligned}
$$


and

$$
\varphi\left(r, X_{r+}^{t, x ; v, \xi}\right)-\varphi\left(r, X_{r}^{t, x ; v, \xi}\right) \geq-K \Delta \xi_{r}
$$

we have

$$
\begin{aligned}
\varphi\left(s, X_{s}^{t, x ; v, \xi}\right) \leq & \varphi\left(t+\delta, X_{t+\delta}^{t, x ; v, \xi}\right) \\
& -\int_{s}^{t+\delta} \frac{\partial}{\partial s} \varphi(r, x)+\mathcal{L}\left(r, X_{r}^{t, x ; v, \xi}, v_{r}\right) \varphi\left(r, X_{r}^{t, x ; v, \xi}\right) \\
& -\int_{s}^{t+\delta} \nabla \varphi\left(r, X_{r}^{t, x ; v, \xi}\right) \sigma\left(r, X_{r}^{t, x ; v, \xi}, v_{r}\right) \mathrm{d} W_{r} \\
& +\int_{s}^{t+\delta} K \mathrm{~d} \xi_{r},
\end{aligned}
$$

only when $\xi_{r} \equiv 0$ above takes equality. For simplicity, putting $\xi \equiv 0$, from (42) and (43), we can get

$$
\begin{aligned}
Y_{s}^{v, 0}-\varphi\left(s, X_{s}^{t, x ; v, 0}\right)= & \int_{s}^{t+\delta}\left[f\left(r, X_{r}^{t, x ; v, 0}, Y_{r}^{v, 0}, Z_{r}^{v, 0}, v_{r}\right)\right. \\
& \left.+\frac{\partial}{\partial s} \varphi(r, x)+\mathcal{L}\left(r, X_{r}^{t, x ; v, 0}, v_{r}\right) \varphi\left(r, X_{r}^{t, x ; v, 0}\right)\right] \mathrm{d} r \\
& -\int_{s}^{t+\delta}\left[Z_{r}^{v, \xi}-\nabla \varphi\left(r, X_{r}^{t, x ; v, 0}\right) \sigma\left(r, X_{r}^{t, x ; v, 0}, v_{r}\right)\right] \mathrm{d} W_{r},
\end{aligned}
$$

which means that

$$
\begin{aligned}
Y_{s}^{1, v, 0} & =Y_{s}^{v, 0}-\varphi\left(s, X_{s}^{t, x ; v, 0}\right) \\
& =\mathcal{G}_{s, t+\delta}^{t, x ; v, 0}\left[\varphi\left(t+\delta, X_{t+\delta}^{t, x ; v, 0}\right)\right]-\varphi\left(s, X_{s}^{t, x ; v, 0}\right), t \leq s \leq t+\delta .
\end{aligned}
$$

Note that in the coefficient $F$ in Eq. (41), it contains the stochastic term $X_{s}^{t, x ; v, \xi}$. We want to take replace it by $x \in \mathbb{R}^{n}$. So we consider the following BSDE also on interval $[t, t+\delta]$ with terminal value 0 ,

$$
Y_{s}^{2, v}=\int_{s}^{t+\delta} F\left(s, x, Y_{s}^{2, v}, Z_{s}^{2, v}, v_{s}\right) \mathrm{d} s-\int_{s}^{t+\delta} Z_{s}^{2, v} \mathrm{~d} W_{s},
$$

where $v \in \mathcal{U}_{1}$. From Lemma 26] (see in Appendix), we have

$$
\left|Y_{s}^{1, v, 0}-Y_{s}^{2, v}\right| \leq C \delta^{\frac{3}{2}}, t \leq s \leq t+\delta .
$$


By virtue of DPP (see Lemma 9), we have

$$
\varphi(t, x)=u(t, x)=e s s \inf _{(v, \xi) \in \mathcal{U}} \mathcal{G}_{t, t+\delta}^{t, x ; v, \xi}\left[u\left(t+\delta, X_{t+\delta}^{t, x ; v, \xi}\right)\right], 0 \leq \delta \leq T-t .
$$

From $u \leq \varphi$ and the monotonicity property of $G_{t, t+\delta}^{t, x ; v, \xi}[\cdot]$, it follows

$$
\begin{aligned}
\varphi(t, x) & =u(t, x) \\
& =e s s \inf _{(v, \xi) \in \mathcal{U}} \mathcal{G}_{t, t+\delta}^{t, x ; v, \xi}\left[u\left(t+\delta, X_{t+\delta}^{t, x ; v, \xi}\right)\right] \\
& \leq e s s \inf _{(v, \xi) \in \mathcal{U}} \mathcal{G}_{t, t+\delta}^{t, x ; v, \xi}\left[\varphi\left(t+\delta, X_{t+\delta}^{t, x ; v, \xi}\right)\right] \\
& \leq e s s \inf _{v \in \mathcal{U}_{1}} \mathcal{G}_{t, t+\delta}^{t, x ; v, 0}\left[\varphi\left(t+\delta, X_{t+\delta}^{t, x ; v, 0}\right)\right],
\end{aligned}
$$

which yields

$$
\text { ess } \inf _{v \in \mathcal{U}_{1}} Y_{t}^{1, v, 0} \geq 0
$$

From (45), we get

$$
Y_{t}^{2, v} \geq Y_{t}^{1, v, 0}-C \delta^{\frac{3}{2}}
$$

Combining to (46) and (47), we must have

$$
Y_{t}^{2, v} \geq-C \delta^{\frac{3}{2}}
$$

Observe that in Eq. (44), $F$ depends on control $v$. Now, eliminate the control term via introducing the following BSDE:

$$
\mathcal{Y}_{s}=\int_{s}^{t+\delta} F_{0}\left(s, x, \mathcal{Y}_{s}, \mathcal{Z}_{s}\right) \mathrm{d} s-\int_{s}^{t+\delta} \mathcal{Z}_{s} \mathrm{~d} W_{s},
$$

where

$$
F_{0}(s, x, y, z)=\inf _{v \in U} F(s, x, y, z, v) .
$$

Clearly,

$$
F(s, x, y, z, v) \geq F_{0}(s, x, y, z),
$$

where $(s, x, y, z, v) \in[0, T] \times \mathbb{R}^{n} \times \mathbb{R} \times \mathbb{R}^{d} \times U$. Applying the comparison theorem (Lemma 4) to Eq. (44) and (49), we have

$$
\text { ess } \inf _{v \in \mathcal{U}_{1}} Y_{t}^{2, v} \geq \mathcal{Y}_{t}, \text { a.s.. }
$$


Indeed, from (51) and Lemma 4, it follows that $Y_{t}^{2, v} \geq \mathcal{Y}_{t}$, a.s. for any $v \in \mathcal{U}_{1}$. Taking essinf, we get (52) immediately. Besides, there exists a measurable function $v^{\prime}:[t, T] \times \mathbb{R}^{n} \times \mathbb{R} \times \mathbb{R}^{d} \rightarrow U$ such that

$F_{0}(s, x, y, z)=F\left(s, x, y, z, v^{\prime}(s, x, y, z)\right), \quad(s, x, y, z) \in[0, T] \times \mathbb{R}^{n} \times \mathbb{R} \times \mathbb{R}^{d}$.

Then taking $v_{s}^{\prime}:=v^{\prime}\left(s, x, \mathcal{Y}_{s}, \mathcal{Z}_{s}\right), t \leq s \leq t+\delta$, we have $v^{\prime} \in \mathcal{U}_{1}$ and

$$
F_{0}\left(s, x, \mathcal{Y}_{s}, \mathcal{Z}_{s}\right)=F\left(s, x, \mathcal{Y}_{s}, \mathcal{Z}_{s}, v_{s}^{\prime}\right), t \leq s \leq t+\delta
$$

According to the uniqueness solution of BSDE (44) and (49), it follows that $\left(\mathcal{Y}_{s}, \mathcal{Z}_{s}\right)=\left(Y_{s}^{2, v^{\prime}}, Z_{s}^{2, v^{\prime}}\right)$. Particularly,

$$
\mathcal{Y}_{t}=Y_{t}^{2, v^{\prime}}, \text { a.s. }
$$

From (52) and (53), it follows that ess $\inf _{v \times \mathcal{U}_{1}} Y_{t}^{2, v}=\mathcal{Y}_{t}$. Then (49) becomes

$$
\mathcal{Y}_{s}^{0}=\int_{s}^{t+\delta} F_{0}\left(s, x, \mathcal{Y}_{s}^{0}, \mathcal{Z}_{s}^{0}\right) \mathrm{d} s-\int_{s}^{t+\delta} \mathcal{Z}_{s}^{0} \mathrm{~d} W_{s} .
$$

Moreover, from the definition of $F_{0}$, it follows that $F_{0}(s, x, y, z)$ is deterministic function. Note that the terminal value is 0 , we immediately get the solution to Eq. (54) is a triple $\left(\mathcal{Y}_{s}^{0}, \mathcal{Z}_{s}^{0}\right)=\left(\overline{\mathcal{Y}}_{s}^{0}, 0\right)$, where $\overline{\mathcal{Y}}_{s}^{0}$ satisfies the following ODE:

$$
\overline{\mathcal{Y}}_{t}^{0}=\int_{t}^{t+\delta} F_{0}\left(s, x, \overline{\mathcal{Y}}_{s}^{0}, 0\right) \mathrm{d} s .
$$

On the other hand, (48) implies that $\int_{t}^{t+\delta} F_{0}\left(s, x, \overline{\mathcal{Y}}_{s}^{0}, 0\right) \mathrm{d} s \geq-C \delta^{\frac{3}{2}}$. By classical estimate, we obtain $F_{0}(s, x, 0,0) \geq 0$, that is

$$
\begin{aligned}
0 \leq & F_{0}(s, x, 0,0) \\
= & \inf _{v \in U} F(s, x, 0,0, v) \\
= & \frac{\partial \varphi}{\partial t}(t, x) \\
& +\min _{v \in U} \mathcal{L}(t, x, v) \varphi(t, x)+f(t, x, \varphi(t, x), \nabla \varphi(t, x) \sigma(t, x, v), v),
\end{aligned}
$$

which leads contradiction to (38).

Next we show that $u$ is meanwhile a supersolution of (30). Suppose that $\varphi \in C^{1,2}\left([0, T] \times \mathbb{R}^{n}\right)$, for any $(t, x) \in[0, T] \times \mathbb{R}^{n}$ such that $u-\varphi$ attains a 
global minimum at $(t, x)$. Without loss of generality we may also suppose that $u(t, x)=\varphi(t, x)$, then

$$
u(\bar{t}, \bar{x})-u(t, x) \geq \varphi(\bar{t}, \bar{x})-\varphi(t, x), \quad(t, x) \neq(\bar{t}, \bar{x}) \in[0, T] \times \mathbb{R}^{n} .
$$

If (36) fails, the following inequity holds true simultaneously,

$$
\begin{gathered}
\frac{\partial \varphi}{\partial t}(t, x)+\min _{v \in U} \mathcal{L}(t, x, v) \varphi(t, x)+f(t, x, \varphi(t, x), \nabla \varphi(t, x) \sigma(t, x, v), v)>0, \\
\varphi_{x}^{\top}(t, x) G_{t}+K>0, \text { for } i, 1 \leq i \leq m .
\end{gathered}
$$

For small $h \in \mathbb{R}_{+}^{m}, h \neq 0, \varphi(t, x+G h)-\varphi(t, x) \geq-K h$. Thus, by (56), $u(t, x+G h)-u(t, x) \geq-K h$, which means $(r, x) \in \mathcal{D}(u)$, and by Lemma 16. we know that $\hat{X}^{t, x ; \hat{v}, \hat{\xi}}$ is continuous for optimal controls $(\hat{v}, \hat{\xi}) \in \mathcal{U}$. Hence, applying Itô's formula to $\varphi(t, x)$, we have

$$
\begin{aligned}
\varphi\left(s, X_{s}^{t, x ; \hat{v}, \hat{\xi}}\right)= & \varphi\left(t+\delta, X_{t+\delta}^{t, x ; \hat{v}, \hat{\xi}}\right) \\
& -\int_{s}^{t+\delta}\left[\frac{\partial}{\partial s} \varphi(r, x)+\mathcal{L}\left(r, X_{r}^{t, x ; \hat{v}, \hat{\xi}}, \hat{v}_{r}\right) \varphi\left(r, X_{r}^{t, x ; \hat{v}, \hat{\xi}}\right)\right] \mathrm{d} r \\
& -\int_{s}^{t+\delta} \nabla \varphi^{\top}\left(r, X_{r}^{t, x ; \hat{v}, \hat{\xi}}\right) G \mathrm{~d} \hat{\xi}_{r} \\
& -\int_{s}^{t+\delta} \nabla \varphi\left(r, X_{r}^{t, x ; \hat{v}, \hat{\xi}}\right) \sigma\left(r, X_{r}^{t, x ; \hat{v}, \hat{\xi}}, \hat{v}_{r}\right) \mathrm{d} W_{r} .
\end{aligned}
$$

Repeating the method above, we introduce the following two BSDEs:

$$
\begin{aligned}
Y_{s}^{\hat{v}, \hat{\xi}}= & \varphi\left(t+\delta, X_{t+\delta}^{t, x ; \hat{v}, \hat{\xi}}\right) \\
& +\int_{s}^{t+\delta} f\left(r, X_{r}^{t, x ; \hat{v}, \hat{\xi}}, Y_{r}^{\hat{v}, \hat{\xi}}, Z_{r}^{\hat{v}, \hat{\xi}}, \hat{v}_{r}\right) \mathrm{d} r \\
& -\int_{s}^{t+\delta} Z_{r}^{\hat{v}, \hat{\xi}} \mathrm{d} W_{s}+\int_{s}^{t+\delta} K \mathrm{~d} \hat{\xi}_{r}
\end{aligned}
$$

and

$$
\begin{aligned}
Y_{s}^{1, \hat{v}, \hat{\xi}}= & \int_{s}^{t+\delta} F\left(s, X_{s}^{t, x ; \hat{v}, \hat{\xi}}, Y_{s}^{1, \hat{v}, \hat{\xi}}, Z_{s}^{1, \hat{v}, \hat{\xi}}, \hat{v}_{s}\right) \mathrm{d} s-\int_{s}^{t+\delta} Z_{s}^{1, \hat{v}, \hat{\xi}} \mathrm{d} W_{s} \\
& +\int_{s}^{t+\delta}\left(K+\nabla \varphi^{\top}\left(r, X_{r}^{t, x ; \hat{v}, \hat{\xi}}\right) G_{r}\right) \mathrm{d} \hat{\xi}_{r} .
\end{aligned}
$$


Clearly,

$$
Y_{s}^{1, \hat{v}, \hat{\xi}}=Y_{s}^{\hat{v}, \hat{\xi}}-\varphi\left(s, X_{s}^{t, x ; \hat{v}, \hat{\xi}}\right) .
$$

Consider the following BSDE:

$$
\begin{aligned}
Y_{s}^{2, \hat{v}, \hat{\xi}}= & \int_{s}^{t+\delta} F\left(s, x, Y_{s}^{2, \hat{v}, \hat{\xi}}, Z_{s}^{2, \hat{v}, \hat{\xi}}, \hat{v}_{s}\right) \mathrm{d} s-\int_{s}^{t+\delta} Z_{s}^{2, \hat{v}, \hat{\xi}} \mathrm{d} W_{s} \\
& +\int_{s}^{t+\delta}\left(K+\nabla \varphi^{\top}\left(r, X_{r}^{t, x ; \hat{v}, \hat{\xi}}\right) G_{r}\right) \mathrm{d} \hat{\xi}_{r} .
\end{aligned}
$$

From Lemma 8 (see in Appendix), we have

$$
\left|Y_{s}^{1, \hat{v}, \hat{\xi}}-Y_{s}^{2, \hat{v}, \hat{\xi}}\right| \leq C \delta^{\frac{3}{2}}, t \leq s \leq t+\delta
$$

which implies that

$$
0 \leq Y_{t}^{2, \hat{v}, \hat{\xi}}-C \delta^{\frac{3}{2}} \leq Y_{t}^{1, \hat{v}, \hat{\xi}} .
$$

Now we give the following BSDE:

$$
\begin{aligned}
\mathcal{Y}_{s}^{0, \hat{\xi}}= & \int_{s}^{t+\delta} F_{0}\left(s, x, \mathcal{Y}_{s}^{0, \hat{\xi}}, \mathcal{Z}_{s}^{0, \hat{\xi}}\right) \mathrm{d} s-\int_{s}^{t+\delta} \mathcal{Z}_{s}^{0, \hat{\xi}} \mathrm{d} W_{s} \\
& +\int_{s}^{t+\delta}\left(K+\nabla \varphi^{\top}\left(r, X_{r}^{t, x ; \hat{v}, \hat{\xi}}\right) G\right) \mathrm{d} \hat{\xi}_{r},
\end{aligned}
$$

where $F_{0}(s, x, y, z)$ is defined in (50). By comprison theorem (Lemma 44), we have $\mathcal{Y}_{s}^{0, \hat{\xi}} \leq Y_{s}^{2, \hat{v}, \hat{\xi}}$, a.s. $s \leq t \leq T$. Since $\hat{v} \in \mathcal{U}_{1}$, we can find $\bar{v}(s, x, y, z) \in$ $\arg \min _{v \in U} F(s, x, y, z, v)$, such that $\hat{v}_{s}=\bar{v}\left(s, X_{r}^{t, x ; \hat{v}, \hat{\xi}}, Y_{r}^{t, x ; \hat{v}, \hat{\xi}}, Z_{r}^{t, x ; \hat{v}, \hat{\xi}}\right)$ and $F_{0}\left(s, x, \mathcal{Y}_{s}^{0, \hat{\xi}}, \mathcal{Z}_{s}^{0, \hat{\xi}}\right)=F\left(s, x, \mathcal{Y}_{s}^{0, \hat{\xi}}, \mathcal{Z}_{s}^{0, \hat{\xi}}, \hat{v}_{s}\right), s \in[t, T]$. Therefore, $\mathcal{Y}_{s}^{0, \hat{\xi}}=$ $Y_{s}^{2, \hat{v}, \hat{\xi}}$, a.s. $s \leq t \leq T$. Particularly, $\mathcal{Y}_{t}^{0, \hat{\xi}}=Y_{t}^{2, \hat{v}, \hat{\xi}}$, a.s. From (65) $0<\mathcal{Y}_{t}^{0, \hat{\xi}}-C \delta^{\frac{3}{2}} \leq Y_{t}^{1, \hat{v}, \hat{\xi}}$. Observing (58), we have $\mathcal{Y}_{t}^{0,0} \leq \mathcal{Y}_{t}^{0, \hat{\xi}}$, where $\mathcal{Y}_{t}^{0,0}$ satisfies

$$
\mathcal{Y}_{s}^{0,0}=\int_{s}^{t+\delta} F_{0}\left(s, x, \mathcal{Y}_{s}^{0,0}, \mathcal{Z}_{s}^{0,0}\right) \mathrm{d} s-\int_{s}^{t+\delta} \mathcal{Z}_{s}^{0,0} \mathrm{~d} W_{s} .
$$

Eq.(67) admits a unique adapted solution $\left(\mathcal{Y}_{s}^{0,0}, \mathcal{Z}_{s}^{0,0}\right)=\left(\tilde{\mathcal{Y}}_{s}^{0}, 0\right)$, where $\tilde{\mathcal{Y}}_{s}^{0}$ satisfies $\tilde{\mathcal{Y}}_{t}^{0}=\int_{t}^{t+\delta} F_{0}\left(s, x, \tilde{\mathcal{Y}}_{s}^{0}, 0\right) \mathrm{d} s$. Hence, we have $0<\tilde{\mathcal{Y}}_{s}^{0}-C \delta^{\frac{3}{2}} \leq Y_{t}^{1, \hat{v}, \hat{\xi}}$. 
From (57), we have $Y_{t}^{1, \hat{v}, \hat{\xi}}>0$, which contradicts the dynamic programming principle. Indeed,

$$
\begin{aligned}
\varphi(t, x) & =u(t, x) \\
& =\mathcal{G}_{t, t+\delta}^{t, x ; \hat{v}, \hat{\xi}}\left[u\left(t+\delta, X_{t+\delta}^{t, x ; \hat{v}, \hat{\xi}}\right)\right] \\
& \geq \mathcal{G}_{t, t+\delta}^{t, x ; \hat{v}, \hat{\xi}}\left[\varphi\left(t+\delta, X_{t+\delta}^{t, x ; \hat{v}, \hat{\xi}}\right)\right],
\end{aligned}
$$

which means that $Y_{t}^{1, \hat{v}, \hat{\xi}} \leq 0$. The proof is complete.

In order to establish a uniqueness result for viscosity solution of (피), we adapt some techniques and methods from [31]. We have to mention that there is another approach developed by Barles, Buckdahn, and Pardoux [7]. The value function can be considered in given class of continuous functions satisfying

$$
\lim _{|x| \rightarrow \infty}|u(t, x)| \exp \left\{-A[\log (|x|)]^{2}\right\}=0,
$$

uniformly for $t \in[0, T]$, for some $A>0$. These techniques and methods can also be found in [13, 64, 23] for the uniqueness for viscosity solutions of recursive control of the obstacle constraint problem and Hamilton-Jacobi-BellmanIsaacs equations related to stochastic differential games, respectively.

However, as you may have observed, in our H-J-B inequality, there appears a term $u_{x}^{\top}(t, x) G+K$, which is a row vector (in contrast to [64], scalar there). It is worth to pointing out that the smooth supersolution built in [7], namely

$$
\chi(t, x)=\exp [(\check{C}(T-t)+A) \psi(x)]
$$

whilst

$$
\psi(x)=\left[\log \left(|x|^{2}+1\right)^{\frac{1}{2}}+1\right]^{2},
$$

where $\check{C}$ and $A$ are positive constants has some properties. After some tedious computations, we have

$$
\chi_{x}(t, x)=2 \chi(t, x)(\check{C}(T-t)+A) \cdot\left(|x|^{2}+1\right)^{-\frac{3}{2}} x .
$$

It follows

$$
\chi_{x}^{T}(t, x) G=2 \chi(t, x)(\check{C}(T-t)+A) \cdot\left(|x|^{2}+1\right)^{-\frac{3}{2}} x^{\top} \cdot G .
$$

Obviously, for any $x \in \mathbb{R}^{n}$, it is impossible to get $x^{\top} \cdot G \geq 0$, unless selecting $x$ such that $x^{\top} \cdot G \geq 0$. 
Consider the following

$$
\left\{\begin{array}{l}
\min \left(w_{x}^{\top}(t, x) G\right. \\
\left.\frac{\partial w}{\partial t}(t, x)+\min _{v \in U}\{\mathcal{L}(t, x, v) w(t, x)+L|w|+L|\nabla w \sigma(t, x, v)|\}\right)=0 \\
w(T, x)=0,0 \leq t \leq T
\end{array}\right.
$$

On the one hand, repeating the method in [7], one can show

$$
\min _{v \in U}\{\mathcal{L}(t, x, v) \chi(t, x)+C|\chi|+C|\nabla \chi \sigma(t, x, v)|\} \leq 0,
$$

where $C$ is the Lipschitz constant of $f$. On the other hand, when choosing $x \in \mathcal{A}:=\left\{x: x^{\top} \cdot G \geq 0\right\}, \chi$ is indeed a supersolution of (68) according to (ii) of Definition [18. However, following the idea in [7], whenever considering the difference of $u_{1}-u_{2}$ where $u_{1}\left(u_{2}\right)$ is a subsolution (supersolution) of (30). The domain of $u_{1}-u_{2}$ must be restricted in $\mathcal{A}$. Hence, we borrow the idea from Fleming, Soner [31] and Haussmann, Suo [36] to handle the uniqueness.

For our aim, let us define $\Sigma=[0, T] \times \mathbb{R}^{n}$ and $C(\Sigma ; \mathbb{R})$ denote the collection of real-valued continuous functions defined on $\Sigma$, value in $\mathbb{R}$. Now introduce the function space

$$
\begin{aligned}
\mathcal{C}(\Sigma):= & \{u: u \in C(\Sigma ; \mathbb{R}), \text { with } u \text { bounded, and } \\
& |u(t, x)-u(t, y) \leq C| x-y|| \text { for some } C>0\} .
\end{aligned}
$$

We now give the uniqueness result for (30). This result is attained under more restrictive assumptions that the existence one. We add the following two additional assumptions:

(A4) Assume that $b, \sigma, \Phi, f(t, \cdot, y, z, v)$ are uniformly continuous, uniformly with respect to $(t, y, z, v)$, and bounded.

(A5) Assume that

$$
|f(t, x, r, p, v)-f(t, x, y, p, v)| \leq \varpi_{R}(|x-y|(1+|p|))
$$

where $\varpi_{R}(s) \rightarrow 0$, when $s \rightarrow 0^{+}$, for all $t \in[0, T],|x|,|y| \leq R$, $|r| \leq R, \forall R>0$. 
We have put somewhat strong assumptions, namely, $b, \sigma, f$ are bounded. These conditions may be removed by modifying the idea by Ishii [37].

Theorem 21. Assume that (A1)-(A5) are in force. Then there exists at most one viscosity solution of $H-J-B$ inequality (30) in the class of bounded and continuous functions.

Proof. Under the assumptions (A1)-(A4), by Proposition 2.5 in [7] and Lemma 10, one can show that the value function $u$ is uniformly continuous and bounded. Thus, $u$ (defined by (9) $) \in \mathcal{C}(\Sigma)$.

Let $u_{1}$ and $u_{2}$ be two viscosity solution to (30). We will show that $u_{1} \leq u_{2}$ on $\Sigma$. For any given $\epsilon: 0<\epsilon<1$, define $\left.\left.\Sigma_{\epsilon}:=\right] \epsilon, T\right] \times \mathbb{R}^{n}$. For any $(t, x) \in \Sigma_{\epsilon}$,

$$
u_{1}^{\epsilon}(t, x)=(1-\epsilon) u_{1}(t, x)-\frac{\epsilon}{1-\epsilon} .
$$

It is easy to check that $\frac{1}{1-\epsilon} u_{1}^{\epsilon}$ is a viscosity subsolution to (30). Given any $\alpha, \beta>0,0<\epsilon<1$, define an auxiliary function

$$
\Psi(t, x, y)=u_{1}^{\epsilon}(t, x)-u_{2}(t, y)-\frac{1}{2 \alpha}|x-y|^{2}+\beta(t-T) .
$$

Clearly, $\Psi$ is bounded above on $\Sigma_{\epsilon}$. We can find $\left(t_{\alpha}, x_{\alpha}, y_{\alpha}\right) \in \Sigma_{\epsilon}$ such that

$$
\Psi\left(t_{\alpha}, x_{\alpha}, y_{\alpha}\right)>\sup _{\Sigma_{\epsilon}} \Psi(t, x, y)-\alpha .
$$

Set

$$
\Psi_{\alpha}(t, x, y)=\Psi(t, x, y)-\frac{1}{2 \alpha}\left[\left|t-t_{\alpha}\right|^{2}+\left|x-x_{\alpha}\right|^{2}+\left|y-y_{\alpha}\right|^{2}\right] .
$$

Clearly, $\Psi_{\alpha}(t, x, y)$ attains the maximum on $\Sigma_{\epsilon}$ at certain point $\left(t^{*}, x^{*}, y^{*}\right)$, which depends implicitly on $\alpha$ and $\beta$. One can immediately get

$$
\left|t^{*}-t_{\alpha}\right|^{2}+\left|x^{*}-x_{\alpha}\right|^{2}+\left|y^{*}-y_{\alpha}\right|^{2} \leq 2 \text {. }
$$

By the method applied in [31], we can show that, for any given $\epsilon>0$,

$$
\left|x^{*}-y^{*}\right|^{2}=o(\alpha) \text {. }
$$

Now we assume that the maximum of the function $\Psi_{\alpha}(t, x, y)$ is always at the point $\left(T, x^{*}, y^{*}\right)$. Then, we have

$$
\Psi_{\alpha}(T, x, y) \leq \Psi_{\alpha}\left(T, x^{*}, y^{*}\right), \forall x, y \in \mathbb{R}^{n} .
$$


Thus

$$
\begin{aligned}
\limsup _{\alpha \rightarrow 0}\left(u_{1}^{\epsilon}\left(T, x^{*}\right)-u_{2}\left(T, y^{*}\right)\right) \leq & \limsup _{\alpha \rightarrow 0}\left((1-\epsilon) u_{1}\left(T, x^{*}\right)-u_{2}\left(T, y^{*}\right)\right) \\
\leq & (1-\epsilon) \limsup _{\alpha \rightarrow 0}\left(\Phi\left(x^{*}\right)-\Phi\left(y^{*}\right)\right) \\
& -\epsilon \limsup _{\alpha \rightarrow 0}\left(y^{*}\right) \\
\leq & \epsilon{ }^{,}
\end{aligned}
$$

since $\Phi$ satisfies Lipschitz condition and (71). The constant $C$ will change line to line. Next, we deal with

$$
\begin{aligned}
& u_{1}^{\epsilon}(t, x)-u_{2}(t, y)+\beta(t-T) \\
= & \Psi(t, x, x) \\
\leq & \limsup _{\epsilon<t<T, x, y \in \mathbb{R}^{n}} \Psi(t, x, y) \\
= & \lim _{\alpha \rightarrow 0} \Psi_{\alpha}\left(t_{\alpha}, x_{\alpha}, y_{\alpha}\right) \\
\leq & \limsup _{\alpha \rightarrow 0} \Psi_{\alpha}\left(T, x^{*}, y^{*}\right) \\
\leq & \limsup _{\alpha \rightarrow 0}\left(u_{1}^{\epsilon}\left(T, x^{*}\right)-u_{2}\left(T, y^{*}\right)\right) \\
\leq & \epsilon C .
\end{aligned}
$$

Here we have used the fact (69). Hence, we have by the definition of $u_{1}^{\epsilon}$

$$
\begin{aligned}
u_{1}(t, x)-u_{2}(t, x) & =\frac{1}{1-\epsilon} u_{1}^{\epsilon}(t, x)-u_{2}(t, x)+\frac{\epsilon}{(1-\epsilon)(t-\epsilon)} \\
& =u_{1}^{\epsilon}(t, x)-u_{2}(t, x)+\frac{\epsilon}{1-\epsilon} u_{1}^{\epsilon}(t, x)+\frac{\epsilon}{(1-\epsilon)(t-\epsilon)} \\
& \leq \epsilon C+\frac{\epsilon}{1-\epsilon} u_{1}^{\epsilon}(t, x)+\frac{\epsilon}{(1-\epsilon)(t-\epsilon)}+\beta(T-t) .
\end{aligned}
$$

Letting $\epsilon, \beta \rightarrow 0$, we obtain

$$
u_{1}(t, x) \leq u_{2}(t, x), \quad(t, x) \in[0, T] \times \mathbb{R}^{n} .
$$

From the arbitrary of $(t, x)$, we conclude that (72) holds on $\Sigma$. Repeating the method above, we are able to get

$$
u_{2}(t, x) \leq u_{1}(t, x), \quad(t, x) \in[0, T] \times \mathbb{R}^{n} .
$$


Apparently, the rest proof will focus on the observation $t^{*}=T$ whenever $\epsilon, \alpha$ are small enough and $\beta>0$. We will prove this result by a contradiction. Suppose that $t^{*}<T$.

Define

$\phi(t, x, y)=\frac{1}{2 \alpha}|x-y|^{2}+\frac{\alpha}{2}\left[\left|t-t_{\alpha}\right|^{2}+\left|x-x_{\alpha}\right|^{2}+\left|y-y_{\alpha}\right|^{2}\right]-\beta T(t-T)$.

By virtue of Theorem 8.3 in [20], there exist $c_{i} \in \mathbb{R}, X_{i} \in \mathbb{S}^{n}, i=1,2$ such that

$$
\text { (i) } \begin{aligned}
\left(c_{1}, D_{x_{1}} \phi\left(t^{*}, x^{*}, y^{*}\right), X_{1}\right) & \in \mathcal{P}^{2,+} u_{1}(t, x), \\
\left(c_{2}, D_{x_{2}} \phi\left(t^{*}, x^{*}, y^{*}\right), X_{2}\right) & \in \mathcal{P}^{2,+}\left(-u_{2}(t, x)\right), \\
(\mathrm{ii})-\left(\frac{1}{\epsilon}+\|A\|\right) I & \leq\left(\begin{array}{cc}
X_{1} & 0 \\
0 & X_{2}
\end{array}\right) \leq A+\epsilon A^{2}, \\
\text { (iii) } c_{1}+c_{2} & =\phi_{t}\left(t^{*}, x^{*}, y^{*}\right),
\end{aligned}
$$

where $A=D_{x}^{2} \phi\left(t^{*}, x^{*}, y^{*}\right)$ with appropriate norm2 2 . From the definition of $\phi$, it is easy to show that

$$
\begin{aligned}
\phi_{t}\left(t^{*}, x^{*}, y^{*}\right) & =-T \beta+\alpha\left(t^{*}-t_{\alpha}\right), \\
D_{x} \phi\left(t^{*}, x^{*}, y^{*}\right) & =\frac{1}{\alpha}\left(x^{*}-y^{*}\right)+\alpha\left(x^{*}-x_{\alpha}\right), \\
D_{y} \phi\left(t^{*}, x^{*}, y^{*}\right) & =-\frac{1}{\alpha}\left(x^{*}-y^{*}\right)+\alpha\left(y^{*}-y_{\alpha}\right), \\
D_{x x}^{2} \phi\left(t^{*}, x^{*}, y^{*}\right) & =\left(\frac{1}{\alpha}+\alpha\right) I, D_{x y}^{2} \phi\left(t^{*}, x^{*}, y^{*}\right)=-\frac{1}{\alpha} I, \\
D_{y y}^{2} \phi\left(t^{*}, x^{*}, y^{*}\right) & =\left(\frac{1}{\alpha}+\alpha\right) I .
\end{aligned}
$$

Clearly, (iii) in (76) can be expressed as

$$
c_{1}+c_{2}=-T \beta+\alpha\left(t^{*}-t_{\alpha}\right)
$$

\footnotetext{
${ }^{2}$ Here, the norm of the symmetric matrix $A$ is

$$
\begin{aligned}
\|A\| & =\sup \{|\lambda|: \lambda \text { is an eigenvalue of } A\} \\
& =\sup \{|\langle A \xi, \xi\rangle|:|\xi| \leq 1\},
\end{aligned}
$$
}

and $I$ denotes the identity matrix. 
and

$$
\begin{aligned}
A & =\left(\begin{array}{cc}
\left(\frac{1}{\alpha}+\alpha\right) I & -\frac{1}{\alpha} I \\
-\frac{1}{\alpha} I & \left(\frac{1}{\alpha}+\alpha\right) I
\end{array}\right) \\
& =\frac{1}{\alpha}\left(\begin{array}{cc}
I & -I \\
-I & I
\end{array}\right)+\alpha\left(\begin{array}{ll}
I & 0 \\
0 & I
\end{array}\right) .
\end{aligned}
$$

Note that $\mathcal{P}^{2,+}\left(-u_{2}(t, x)\right)=-\mathcal{P}^{2,-}\left(u_{2}(t, x)\right)$ and for any $\lambda>0$, we have

$$
\mathcal{P}^{2,+}\left(\lambda u_{2}\right)=\lambda \mathcal{P}^{2,+}\left(u_{2}\right), \mathcal{P}^{2,-}\left(\lambda u_{2}\right)=\lambda \mathcal{P}^{2,-}\left(u_{2}\right)
$$

Then, (i) in (74) can be rewritten as

$$
\begin{aligned}
\left(\frac{1}{1-\epsilon} c_{1}, \frac{1}{1-\epsilon} D_{x} \phi\left(t^{*}, x^{*}, y^{*}\right), \frac{1}{1-\epsilon} X_{1}\right) & \in \frac{1}{1-\epsilon} \mathcal{P}^{2,+} u_{1}^{\epsilon}(t, x), \\
\left(-c_{2},-D_{y} \phi\left(t^{*}, x^{*}, y^{*}\right),-X_{2}\right) & \in \mathcal{P}^{2,-} u_{2}(t, x)
\end{aligned}
$$

From the Definition [18, it yields

$$
\begin{aligned}
& \min \left(\frac{1}{1-\epsilon} D_{x} \phi\left(t^{*}, x^{*}, y^{*}\right) G+K\right. \\
& \left.\frac{1}{1-\epsilon} c_{1}+\mathcal{H}\left(t^{*}, x^{*}, \frac{1}{1-\epsilon} D_{x} \phi\left(t^{*}, x^{*}, y^{*}\right), \frac{1}{1-\epsilon} X_{1}\right)\right) \geq 0
\end{aligned}
$$

and

$$
\begin{aligned}
& \min \left(-D_{y} \phi\left(t^{*}, x^{*}, y^{*}\right) G+K,\right. \\
& \left.-c_{2}+\mathcal{H}\left(t^{*}, x^{*},-D_{y} \phi\left(t^{*}, x^{*}, y^{*}\right),-X_{2}\right)\right) \leq 0 .
\end{aligned}
$$

Obviously, from (84) we always have

$$
\left\{\begin{array}{l}
\frac{1}{1-\epsilon} D_{x} \phi\left(t^{*}, x^{*}, y^{*}\right) G+K \geq 0 \\
\frac{1}{1-\epsilon} c_{1}+\mathcal{H}\left(t^{*}, x^{*}, \frac{1}{1-\epsilon} D_{x} \phi\left(t^{*}, x^{*}, y^{*}\right), \frac{1}{1-\epsilon} X_{1}\right) \geq 0
\end{array}\right.
$$

We will show that

$$
-D_{y} \phi\left(t^{*}, x^{*}, y^{*}\right) G+K>0 .
$$

Assume that (87) is not true, then for $1 \leq i \leq m$,

$$
-\left(D_{y} \phi\left(t^{*}, x^{*}, y^{*}\right) G\right)^{i}+K^{i}<0,
$$

where $(\eta)^{i}$ stands for the $i$ th coordinate of $\eta$ when $\eta$ is row vector. Now multiply the $i$ th inequality of the first term in (84) by $1-\epsilon$ to get

$$
\left(D_{x} \phi\left(t^{*}, x^{*}, y^{*}\right) G\right)^{i}+(1-\epsilon) K^{i}>0 .
$$


From (88), (89), (78) and (79), we have

$$
\begin{aligned}
\epsilon K^{i} & <\left(D_{x} \phi\left(t^{*}, x^{*}, y^{*}\right)^{\top} G\right)^{i}+\left(D_{y} \phi\left(t^{*}, x^{*}, y^{*}\right)^{\top} G\right)^{i} \\
& =\frac{1}{\alpha}\left(x^{*}-y^{*}\right)+\alpha\left(x^{*}-x_{\alpha}\right)-\frac{1}{\alpha}\left(x^{*}-y^{*}\right)+\alpha\left(y^{*}-y_{\alpha}\right) \\
& =\alpha\left(\left[\left(x^{*}-x_{\alpha}\right)^{\top}+\left(y^{*}-y_{\alpha}\right)^{\top}\right] G\right)^{i} .
\end{aligned}
$$

So when we fix $\epsilon$, and take $\alpha$ small enough, it will lead a contradiction since $K^{i}>k_{0}>0,1 \leq i \leq m$. Therefore, when $\alpha$ is small, (85) is equivalent to

$$
-c_{2}+\mathcal{H}\left(t^{*}, x^{*},-D_{y} \phi\left(t^{*}, x^{*}, y^{*}\right),-X_{2}\right)>0 .
$$

Combing (86) and (90), we get

$$
\begin{aligned}
& c_{1}+(1-\epsilon) \mathcal{H}\left(t^{*}, x^{*}, \frac{1}{1-\epsilon} D_{x} \phi\left(t^{*}, x^{*}, y^{*}\right), \frac{1}{1-\epsilon} X_{1}\right) \\
& +c_{2}-\mathcal{H}\left(t^{*}, x^{*},-D_{y} \phi\left(t^{*}, x^{*}, y^{*}\right),-X_{2}\right) \\
> & 0 .
\end{aligned}
$$

Noting (77), (82) and (91) can be expressed as

$$
\begin{aligned}
& (1-\epsilon) \mathcal{H}\left(t^{*}, x^{*}, \frac{1}{1-\epsilon} D_{x} \phi\left(t^{*}, x^{*}, y^{*}\right), \frac{1}{1-\epsilon} X_{1}\right) \\
& -\mathcal{H}\left(t^{*}, x^{*},-D_{y} \phi\left(t^{*}, x^{*}, y^{*}\right),-X_{2}\right) \\
> & \beta T-\alpha\left(t^{*}-t_{\alpha}\right) .
\end{aligned}
$$

Then, we will estimate (92) step by step. At the beginning,

$$
\begin{aligned}
& (1-\epsilon) \mathcal{H}\left(t^{*}, x^{*}, \frac{1}{1-\epsilon} D_{x} \phi\left(t^{*}, x^{*}, y^{*}\right), \frac{1}{1-\epsilon} X_{1}\right) \\
= & (1-\epsilon) \inf _{v \in U}\left\{\frac{1}{2} \operatorname{Tr}\left(\sigma \sigma^{*}\left(t^{*}, x^{*}, v\right) \frac{1}{1-\epsilon} X_{1}\right)\right. \\
& +\frac{1}{1-\epsilon}\left\langle D_{x} \phi\left(t^{*}, x^{*}, y^{*}\right), b\left(t^{*}, x^{*}, v\right)\right\rangle \\
& \left.+f\left(t^{*}, x^{*}, u_{1}^{\epsilon}\left(t^{*}, x^{*}\right), \frac{1}{1-\epsilon} D_{x} \phi\left(t^{*}, x^{*}, y^{*}\right) \sigma\left(t^{*}, x^{*}, v\right), v\right)\right\},
\end{aligned}
$$


whilst

$$
\begin{aligned}
& \mathcal{H}\left(t^{*}, y^{*},-D_{y} \phi\left(t^{*}, x^{*}, y^{*}\right),-X_{2}\right) \\
= & \inf _{v \in U}\left\{-\frac{1}{2} \operatorname{Tr}\left(\sigma \sigma^{*}\left(t^{*}, y^{*}, v\right) X_{2}\right)+\left\langle-D_{y} \phi\left(t^{*}, x^{*}, y^{*}\right), b\left(t^{*}, y^{*}, v\right)\right\rangle\right. \\
& \left.+f\left(t^{*}, y^{*}, u_{2}\left(t^{*}, y^{*}\right),-D_{y} \phi\left(t^{*}, x^{*}, y^{*}\right) \sigma\left(t^{*}, y^{*}, v\right), v\right)\right\} .
\end{aligned}
$$

Next, (92) can be rewritten as

$$
\begin{aligned}
& (1-\epsilon) \mathcal{H}\left(t^{*}, x^{*}, \frac{1}{1-\epsilon} D_{x} \phi\left(t^{*}, x^{*}, y^{*}\right), \frac{1}{1-\epsilon} X_{1}\right) \\
& -\mathcal{H}\left(t^{*}, x^{*},-D_{y} \phi\left(t^{*}, x^{*}, y^{*}\right),-X_{2}\right) \\
\leq & \sup _{v \in U}\left\{\frac{1}{2} \operatorname{Tr}\left(\sigma \sigma^{*}\left(t^{*}, x^{*}, v\right) X_{1}\right)+\frac{1}{2} \operatorname{Tr}\left(\sigma \sigma^{*}\left(t^{*}, y^{*}, v\right) X_{2}\right)\right. \\
& +\left\langle D_{x} \phi\left(t^{*}, x^{*}, y^{*}\right), b\left(t^{*}, x^{*}, v\right)\right\rangle+\left\langle D_{y} \phi\left(t^{*}, x^{*}, y^{*}\right), b\left(t^{*}, y^{*}, v\right)\right\rangle \\
& +(1-\epsilon) f\left(t^{*}, x^{*}, u_{1}^{\epsilon}\left(t^{*}, x^{*}\right), \frac{1}{1-\epsilon} D_{x} \phi\left(t^{*}, x^{*}, y^{*}\right) \sigma\left(t^{*}, x^{*}, v\right), v\right) \\
& \left.-f\left(t^{*}, y^{*}, u_{2}\left(t^{*}, y^{*}\right),-D_{y} \phi\left(t^{*}, x^{*}, y^{*}\right) \sigma\left(t^{*}, y^{*}, v\right), v\right)\right\} .
\end{aligned}
$$

Set

$$
\begin{aligned}
\Upsilon_{1}= & \frac{1}{2} \operatorname{Tr}\left(\sigma \sigma^{*}\left(t^{*}, x^{*}, v\right) X_{1}\right)+\frac{1}{2} \operatorname{Tr}\left(\sigma \sigma^{*}\left(t^{*}, y^{*}, v\right) X_{2}\right), \\
\Upsilon_{2}= & \left\langle D_{x} \phi\left(t^{*}, x^{*}, y^{*}\right), b\left(t^{*}, x^{*}, v\right)\right\rangle+\left\langle D_{y} \phi\left(t^{*}, x^{*}, y^{*}\right), b\left(t^{*}, y^{*}, v\right)\right\rangle, \\
\Upsilon_{3}= & (1-\epsilon) f\left(t^{*}, x^{*}, u_{1}^{\epsilon}\left(t^{*}, x^{*}\right), \frac{1}{1-\epsilon} D_{x} \phi\left(t^{*}, x^{*}, y^{*}\right) \sigma\left(t^{*}, x^{*}, v\right), v\right) \\
& -f\left(t^{*}, y^{*}, u_{2}\left(t^{*}, y^{*}\right),-D_{y} \phi\left(t^{*}, x^{*}, y^{*}\right) \sigma\left(t^{*}, y^{*}, v\right), v\right) .
\end{aligned}
$$


For $\Upsilon_{1}$, with $\sigma_{1}=\sigma\left(t^{*}, x^{*}, v\right), \sigma_{2}=\sigma\left(t^{*}, y^{*}, v\right)$, from (75) and (83), we have

$$
\begin{aligned}
& \frac{1}{2} \operatorname{Tr}\left(\sigma \sigma^{*}\left(t^{*}, x^{*}, v\right) X_{1}\right)+\frac{1}{2} \operatorname{Tr}\left(\sigma \sigma^{*}\left(t^{*}, y^{*}, v\right) X_{2}\right) \\
= & \frac{1}{2} \operatorname{Tr}\left(\begin{array}{cc}
\sigma_{1} \sigma_{1}^{\top} & \sigma_{1} \sigma_{2}^{\top} \\
\sigma_{2} \sigma_{1}^{\top} & \sigma_{2} \sigma_{2}^{\top}
\end{array}\right)\left(\begin{array}{cc}
X_{1} & 0 \\
0 & X_{2}
\end{array}\right) \\
\leq & \frac{1}{2}\left(\frac{3}{\alpha}+2 \alpha\right)\left\|\sigma_{1}-\sigma_{1}\right\|^{2}+C \frac{1}{2}\left(\alpha+\alpha^{3}\right) \\
\leq & \frac{C^{2}}{2}\left(\frac{3}{\alpha}+2 \alpha\right)\left|x^{*}-y^{*}\right|^{2}+C \frac{1}{2}\left(\alpha+\alpha^{3}\right) \\
\leq & o(1)
\end{aligned}
$$

where we have used the the assumption that $\sigma$ is bounded and Lipschitz condition.

For $\Upsilon_{2}$, we derive

$$
\begin{aligned}
& \left\langle D_{x} \phi\left(t^{*}, x^{*}, y^{*}\right), b\left(t^{*}, x^{*}, v\right)\right\rangle+\left\langle D_{y} \phi\left(t^{*}, x^{*}, y^{*}\right), b\left(t^{*}, y^{*}, v\right)\right\rangle \\
= & \left\langle\frac{1}{\alpha}\left(x^{*}-y^{*}\right)+\alpha\left(x^{*}-x_{\alpha}\right), b\left(t^{*}, x^{*}, v\right)\right\rangle \\
& +\left\langle-\frac{1}{\alpha}\left(x^{*}-y^{*}\right)+\alpha\left(y^{*}-y_{\alpha}\right), b\left(t^{*}, y^{*}, v\right)\right\rangle \\
\leq & \frac{C}{\alpha}\left|x^{*}-y^{*}\right|^{2}+2 C \alpha \\
\leq & o(1) .
\end{aligned}
$$

For $\Upsilon_{3}$, we handle

$$
\begin{aligned}
& (1-\epsilon) f\left(t^{*}, x^{*}, u_{1}^{\epsilon}\left(t^{*}, x^{*}\right), \frac{1}{1-\epsilon} D_{x} \phi\left(t^{*}, x^{*}, y^{*}\right) \sigma\left(t^{*}, x^{*}, v\right), v\right) \\
& -f\left(t^{*}, y^{*}, u_{2}\left(t^{*}, y^{*}\right),-D_{y} \phi\left(t^{*}, x^{*}, y^{*}\right) \sigma\left(t^{*}, y^{*}, v\right)\right) \\
\leq & (1-\epsilon)\left[\varpi\left(\left|x^{*}-y^{*}\right|\left(1+\frac{1}{1-\epsilon}\right) D_{x} \phi\left(t^{*}, x^{*}, y^{*}\right) \sigma\left(t^{*}, x^{*}, v\right)\right)\right. \\
+ & C\left|u_{1}^{\epsilon}\left(t^{*}, x^{*}\right)-u_{2}\left(t^{*}, y^{*}\right)\right| \\
+ & C\left|\frac{1}{1-\epsilon} D_{x} \phi\left(t^{*}, x^{*}, y^{*}\right) \sigma\left(t^{*}, x^{*}, v\right)+D_{y} \phi\left(t^{*}, x^{*}, y^{*}\right) \sigma\left(t^{*}, y^{*}, v\right)\right|,
\end{aligned}
$$


where we have used the Lipschitz continuity of $f$. Now

$$
\begin{aligned}
& \left|\frac{1}{1-\epsilon} D_{x} \phi\left(t^{*}, x^{*}, y^{*}\right) \sigma\left(t^{*}, x^{*}, v\right)+D_{y} \phi\left(t^{*}, x^{*}, y^{*}\right) \sigma\left(t^{*}, y^{*}, v\right)\right| \\
\leq & \frac{1}{\alpha(1-\epsilon)}\left|\left(x^{*}-y^{*}\right)\left(\sigma\left(t^{*}, x^{*}, v\right)-\sigma\left(t^{*}, y^{*}, v\right)\right)\right| \\
& +\frac{1}{1-\epsilon}\left|\alpha\left(x^{*}-x_{\alpha}\right) \sigma\left(t^{*}, x^{*}, v\right)+\alpha\left(y^{*}-y_{\alpha}\right) \sigma\left(t^{*}, y^{*}, v\right)\right| \\
\leq & \frac{\left|x^{*}-y^{*}\right|^{2}}{\alpha(1-\epsilon)}+\frac{2 \alpha C}{1-\epsilon},
\end{aligned}
$$

where we have used the bound of $\sigma$ and the fact (170). So

$$
\begin{aligned}
& (1-\epsilon) f\left(t^{*}, x^{*}, u_{1}^{\epsilon}\left(t^{*}, x^{*}\right), \frac{1}{1-\epsilon} D_{x} \phi\left(t^{*}, x^{*}, y^{*}\right) \sigma\left(t^{*}, x^{*}, v\right), v\right) \\
& -f\left(t^{*}, y^{*}, u_{2}\left(t^{*}, y^{*}\right),-D_{y} \phi\left(t^{*}, x^{*}, y^{*}\right) \sigma\left(t^{*}, y^{*}, v\right), v\right) \\
\leq & (1-\epsilon) \varpi\left(\left|x^{*}-y^{*}\right|\left(1+\frac{1}{1-\epsilon} D_{x} \phi\left(t^{*}, x^{*}, y^{*}\right) \sigma\left(t^{*}, x^{*}, v\right)\right)\right) \\
& +C \epsilon+\beta\left(1-t^{*}\right)+\frac{\left|x^{*}-y^{*}\right|^{2}}{\alpha(1-\epsilon)}+\frac{2 \alpha C}{1-\epsilon} .
\end{aligned}
$$

Now from (93), (94) and (95), we have

$$
\begin{aligned}
& \beta T-\alpha\left(t^{*}-t_{\alpha}\right) \\
\leq & o(1)+(1-\epsilon) \varpi\left(\left|x^{*}-y^{*}\right|\left(1+\frac{1}{1-\epsilon} D_{x} \phi\left(t^{*}, x^{*}, y^{*}\right) \sigma\left(t^{*}, x^{*}, v\right)\right)\right) \\
& +C \epsilon+\beta\left(T-t^{*}\right)+\frac{\left|x^{*}-y^{*}\right|^{2}}{\alpha(1-\epsilon)}+\frac{2 \alpha C}{1-\epsilon} .
\end{aligned}
$$

Letting $\epsilon, \alpha \rightarrow 0$, and noticing $t^{*}<T$, we obtain

$$
\beta t^{*} \leq 0
$$

which leads to a contradiction. Consequently, $t^{*}=T$ holds true. We thus complete the proof.

Remark 22. The present paper considers a stochastic optimal singular control problem, in which the cost function is defined through a backward stochastic differential equation with singular control. It has been shown that the value 
function is a unique viscosity solution of the corresponding Hamilton-JacobiBellman inequality (30), in a given class of continuous and bounded functions. Nonetheless, as an important issue, the existence of optimal singular controls has never been exploited. In Haussmannand, Suo [35], the authors apply the compactification method to study the classical and singular control problem of Itô's type of stochastic differential equation, where the problem is reformulated as a martingale problem on an appropriate canonical space after the relaxed form of the classical control is introduced. Under some mild continuity assumptions on the data, they obtain the existence of optimal control by purely probabilistic arguments. Nevertheless, in the framework of BSDE, the trajectory of $Y$ seems to be a càdlàg process (from French, for right continuous with left hand limits). Hence, we may consider $Y$ in some space with appropriate topologies, for instance, Meyer-Zheng topology and obtain the convergence of probability measures deduced by $Y$ involving relaxed control. For this topic, reader can also refer articles [35, 33] in this direction. We point out that the related work from the technique of PDEs (see [14, 15] therein). From Wang [63], it is possible to construct the optimal control via the existence of diffusion with refections (see [19]). However, it is interesting to expand this result to forward backward stochastic systems. We shall consider these crucial topics in future. As this complete remake of the existence is much longer than the present paper, it will be reported elsewhere.

\section{Example}

In this section, we illustrate our theoretic result by looking at a concrete example.

Example 23. Consider the following controlled $S D E(n=1)$,

$$
\left\{\begin{aligned}
\mathrm{d} X_{s}^{t, x ; v, \xi}= & {\left[X_{s}^{t, x ; v, \xi}+X_{s}^{t, x ; v, \xi} v_{s}\right] \mathrm{d} s+X_{s}^{t, x ; v, \xi} v_{s} \mathrm{~d} W_{s} } \\
& +G \mathrm{~d} \xi_{s}, s \in[t, T] \\
X_{t}^{t, x ; v, \xi}= & x,
\end{aligned}\right.
$$

with the control domain $U=[-1,0] \cup[1,2]$, constants $G>0$ and $K>0$.

The cost functional is defined by

$$
\left\{\begin{array}{l}
\mathrm{d} Y_{s}^{t, x ; v, \xi}=Z_{s}^{t, x ; v, \xi} v_{s} \mathrm{~d} s+Z_{s}^{t, x ; v, \xi} \mathrm{d} W_{s}+K \mathrm{~d} \xi_{s}, s \in[t, T] \\
Y_{T}^{t, x ; v, \xi}=X_{T}^{t, x ; v, \xi}
\end{array}\right.
$$


It is easy to give the associated $H-J-B$ inequality is

$$
\left\{\begin{array}{l}
\min \left\{G_{t} u_{x}+K, u_{t}+x u_{x}+\min _{v \in U}\left[\frac{1}{2} u_{x x} x^{2} v^{2}+x v u_{x}-x v^{2} u_{x}\right]\right\}=0, \\
u(T, x)=x, \text { for all } x \in \mathbb{R}^{n}
\end{array}\right.
$$

By Theorem 21, we deal with the second term in (96), then obtain

$$
u(t, x)= \begin{cases}e^{t-T} x, & \text { if } x>0, \\ e^{T-t} x, & \text { if } x \leq 0 .\end{cases}
$$

Assume that the optimal control is $\left(v^{*}, \xi^{*}\right)$. If $x \leq 0$, then $u\left(s, X^{t, x ; v^{*}, \xi^{*}}(s)\right)=$ $e^{T-s} X^{t, x ; v^{*}, \xi^{*}}(s)$, which yields that

$$
\mathcal{P}^{2,+} u\left(s, X^{t, x ; v^{*}, \xi^{*}}(s)\right)=\left[e^{t-T} x,+\infty\right) \times\left[e^{t-T},+\infty\right) \times[0,+\infty),
$$

and

$$
\mathcal{P}^{2,-} u\left(s, X^{t, x ; v^{*}, \xi^{*}}(s)\right)=\left(-\infty, e^{t-T} x\right] \times\left(-\infty, e^{t-T}\right] \times(-\infty, 0] .
$$

It is fairly easy to verify that, for any $(p, q, X) \in \mathcal{P}^{2,+} u(t, x)$

$$
\begin{aligned}
G q+K & \geq 0, \\
p+\frac{1}{2} X x^{2}-x q & \geq 0,
\end{aligned}
$$

which means that $u(t, x)$ is the subsolution when $x \leq 0$. Moreover, for $(\tilde{p}, \tilde{q}, \tilde{X}) \in \mathcal{P}^{2,-} u(t, x)$, we have

$$
\begin{aligned}
G \tilde{q}+K & \geq 0, \\
\tilde{p}+\frac{1}{2} \tilde{X} x^{2}-x \tilde{q} & \leq 0,
\end{aligned}
$$

which means $u(t, x)$ is the supersolution.

\section{Appendix A. Forward-backward SDEs with Singular Controls}

In this section, we revisit the theory of FBSDEs with controls. We will give some useful estimates for (7). Consider the following SDE with controls by the initial condition $(t, x) \in[0, T] \times \mathbb{R}^{n}$,

$$
\left\{\begin{aligned}
\mathrm{d} X_{s}^{t, x, v, \xi} & =b\left(s, X_{s}^{t, x, v, \xi}, v_{s}\right) \mathrm{d} s+\sigma\left(s, X_{s}^{t, x, v, \xi}, v_{s}\right) \mathrm{d} W_{s}+G_{s} \mathrm{~d} \zeta_{s}, \\
X_{t}^{t, x, v, \xi} & =x, s \in[0, T] .
\end{aligned}\right.
$$


Lemma 24. Assume that (A1)-(A3) hold, SDE (A.1) admits a strong solution, and for any $t \in[0, T]$, and $x, x^{\prime} \in \mathbb{R}^{n}$, there exists positive constant $C>0$ ( $C$ depends only on the Lipschitz and linear growth constant for $b, \sigma$, with respect to $x$ and $u, G$ and $\xi)$,

$$
\begin{aligned}
\mathbb{E}\left[\sup _{t \leq s \leq T}\left|X_{s}^{t, x, v, \xi}-X_{s}^{t, x^{\prime}, v, \xi}\right|^{2}\right] & \leq C\left|x-x^{\prime}\right|^{2}, \\
\mathbb{E}\left[\sup _{t \leq s \leq T}\left|X_{s}^{t, x, v, \xi}\right|^{2}\right] & \leq C\left(1+|x|^{2}\right) .
\end{aligned}
$$

Proof. The estimate (A.2) can be seen in Theorem 4.1 in [36]. Inequality (A.3) can be obtained by the classical approach (Burkholder-Davis-Gundy inequality and Gronwall's Lemma), so we omit the proof.

Lemma 25. Assume that (A1)-(A3) hold. Then for all $0 \leq t \leq T, x$, $x^{\prime} \in \mathbb{R}^{n}$, we have

$$
\begin{gathered}
\mathbb{E}^{\mathscr{F _ { t }}}\left[\sup _{t \leq s \leq T}\left|Y_{s}^{t, x, v, \xi}-Y_{s}^{t, x^{\prime}, v, \xi}\right|^{2}+\int_{s}^{T}\left|Z_{s}^{t, x, v, \xi}-Z_{s}^{t, x^{\prime}, v, \xi}\right|^{2} \mathrm{~d} s\right] \leq C\left|x-x^{\prime}\right| \text {, a.s. } \\
\mathbb{E}^{\mathscr{F _ { t }}}\left[\sup _{t \leq s \leq T}\left|Y_{s}^{t, x, v, \xi}\right|^{2}+\int_{s}^{T}\left|Z_{s}^{t, x, v, \xi}\right|^{2} \mathrm{~d} s\right] \leq C\left(1+|x|^{2}\right) \text {, a.s. } \\
\mathbb{E}^{\mathscr{F} t}\left[\left|X_{t}^{t, x, v, \xi}-X_{t}^{t, x, v^{\prime}, \xi^{\prime}}\right|^{2}\right] \leq C \mathbb{E}^{\mathscr{F} t}\left[\int_{s}^{T}\left|v_{s}-v_{s}^{\prime}\right|^{2} \mathrm{~d} s+\left|\xi_{s}-\xi_{s}^{\prime}\right|^{2}\right], \text { a.s. } \\
\leq \mathbb{E}^{\mathscr{F} t}\left[\left|Y_{t}^{t, x, v, \xi}-Y_{t}^{t, x, v^{\prime}, \xi^{\prime}}\right|^{2}+\int_{t}^{T}\left|Z_{s}^{t, x, v, \xi}-Z_{s}^{t, x, v^{\prime}, \xi^{\prime}}\right|^{2} \mathrm{~d} s\right] \\
\leq C \mathbb{E}^{\mathscr{F _ { t }}}\left[\int_{s}^{T}\left(\left|v_{s}-v_{s}^{\prime}\right|^{2}+\left|\xi_{s}-\xi_{s}^{\prime}\right|^{2}\right) \mathrm{d} s+\left|\xi_{T}-\xi_{T}^{\prime}\right|^{2}\right], \text { a.s. }
\end{gathered}
$$

Proof. Suppose $\left(Y_{s}^{t, x ; v, \xi}, Z_{s}^{t, x ; v, \xi}\right)$ and $\left(Y_{s}^{t, x^{\prime}, v, \xi}, Z_{s}^{t, x^{\prime}, v, \xi}\right)$ are the solutions to the following BSDEs, respectively,

$$
\begin{aligned}
Y_{s}^{t, x ; v, \xi}= & \Phi\left(X_{T}^{t, x ; v, \xi}\right)+\int_{s}^{T} f\left(r, X_{r}^{t, x ; v, \xi}, Y_{r}^{t, x ; v, \xi}, Z_{r}^{t, x ; v, \xi}, v_{r}\right) \mathrm{d} s \\
& -\int_{s}^{T} Z_{r}^{t, x ; v, \xi} \mathrm{d} W_{r}+K \xi_{T}-K \xi_{s},
\end{aligned}
$$




$$
\begin{aligned}
Y_{s}^{t, x^{\prime} ; v, \xi}= & \Phi\left(X_{T}^{t, x^{\prime} ; v, \xi}\right)+\int_{s}^{T} f\left(r, X_{r}^{t, x^{\prime} ; v, \xi}, Y_{r}^{t, x^{\prime} ; v, \xi}, Z_{r}^{t, x^{\prime} ; v, \xi}, v_{r}\right) \mathrm{d} s \\
& -\int_{s}^{T} Z_{r}^{t, x^{\prime} ; v, \xi} \mathrm{d} W_{r}+K \xi_{T}-K \xi_{s}
\end{aligned}
$$

We can get estimate (A.4) by B-D-G inequality and (A.2). Put $\bar{Y}_{s}^{t, x, v, \xi}=$ $Y_{s}^{t, x ; v, \xi}+K \xi_{s}$. Then Eq. (A.8) becomes to

$$
\begin{aligned}
\bar{Y}_{s}^{t, x ; v, \xi}= & \bar{Y}_{T}^{t, x ; v, \xi}+\int_{s}^{T} f\left(t, X_{r}^{t, x ; v, \xi}, \bar{Y}_{r}^{t, x ; v, \xi}-K \xi_{r}, Z_{r}^{t, x ; v, \xi}, v_{r}\right) \mathrm{d} r \\
& -\int_{s}^{T} Z_{r}^{t, x ; v, \xi} \mathrm{d} W_{r} .
\end{aligned}
$$

Applying Itô's formula to $\left|\bar{Y}_{s}^{t, x, v, \xi}\right|^{2}$ on the internal $[s, T]$, we have

$$
\begin{aligned}
& \mathbb{E}\left[\left|Y_{s}^{t, x, v, \xi}\right|^{2}+\frac{1}{2} \int_{s}^{T}\left|Z_{r}^{t, x ; v, \xi}\right|^{2} \mathrm{~d} r\right] \leq \mathbb{E}\left[\left|\bar{Y}_{s}^{t, x, v, \xi}\right|^{2}+\frac{1}{2} \int_{s}^{T}\left|Z_{r}^{t, x ; v, \xi}\right|^{2} \mathrm{~d} r\right] \\
= & \mathbb{E}^{\mathscr{F} t}\left[\left|\bar{Y}_{T}^{t, x, v, \xi}\right|^{2}+2 \int_{s}^{T} \bar{Y}_{r}^{t, x ; v, \xi} f\left(t, X_{r}^{t, x ; v, \xi}, \bar{Y}_{r}^{t, x ; v, \xi}-K \xi_{r}, Z_{r}^{t, x ; v, \xi}, v_{r}\right) \mathrm{d} r\right] \\
\leq & C \mathbb{E}^{\mathscr{F} t}\left[1+|x|^{2}+\left|K \xi_{T}\right|^{2}+\int_{s}^{T}\left|v_{r}\right|^{2} \mathrm{~d} r+\int_{s}^{T}\left|Y_{r}^{t, x ; v, \xi}\right|^{2} \mathrm{~d} r\right] .
\end{aligned}
$$

By the Grownwall inequality, we can get the estimate (A.5).

Set

$$
\begin{aligned}
\tilde{Y}_{s}^{t, v, \xi}: & =Y_{s}^{t, x, v, \xi}-Y_{s}^{t, x^{\prime}, v, \xi} \\
\tilde{Z}_{s}^{t, v, \xi}: & =Z_{s}^{t, x, v, \xi}-Z_{s}^{t, x^{\prime}, v, \xi} \\
\tilde{f}_{r}: & =f\left(r, X_{r}^{t, x ; v, \xi}, Y_{r}^{t, x ; v, \xi}, Z_{r}^{t, x ; v, \xi}, v_{r}\right) \\
& -f\left(r, X_{r}^{t, x^{\prime} ; v, \xi}, Y_{r}^{t, x^{\prime} ; v, \xi}, Z_{r}^{t, x^{\prime} ; v, \xi}, v_{r}\right) .
\end{aligned}
$$

Then, consider the following BSDE:

$$
\tilde{Y}_{s}^{t, v, \xi}=\tilde{Y}_{T}^{t, v, \xi}+\int_{s}^{T} \tilde{f}_{r} \mathrm{~d} r-\int_{s}^{T} \tilde{Z}_{r}^{t, v, \xi} \mathrm{d} W_{r} .
$$

Using Ito's formula to $\left|X_{t}^{t, x, v, \xi}-X_{t}^{t, x, v^{\prime}, \xi^{\prime}}\right|^{2}$ and $\left|Y_{t}^{t, x, v, \xi}-Y_{t}^{t, x, v^{\prime}, \xi^{\prime}}\right|^{2}$, respectively, it is easy to get (A.6) and (A.7). We omit the proof of since its similar to (A.5). 
Lemma 26. Consider the BSDEs (41) and (44). There exists a positive constant $C$ such that, for any $(v, \xi) \in \mathcal{U}$, we have

$$
\left|Y_{t}^{1, v, 0}-Y_{t}^{2, v}\right| \leq C \delta^{\frac{3}{2}}, \text { a.s. }
$$

Proof. By classical estimate to SDE (A.1), we have for some positive constant C

$$
\mathbb{E}^{\mathscr{F} t}\left[\sup _{t \leq s \leq t+\delta}\left|X^{t, x ; v, 0}-x\right|^{2}\right] \leq C \delta\left(1+|x|^{2}\right) .
$$

Applying the Itô's formula to BSDE (41) and (44), we have

$$
\begin{aligned}
& \mathbb{E}^{\mathscr{F _ { t }}}\left[\int_{t}^{t+\delta}\left(\left|Y_{s}^{1, v, 0}-Y_{s}^{2, v}\right|^{2}+\left|Z_{s}^{1, v, 0}-Z_{s}^{2, v}\right|^{2}\right) \mathrm{d} s\right] \\
\leq & C \mathbb{E}^{\mathscr{F _ { t }}}\left[\int_{t}^{t+\delta}\left|X_{s}^{1, v, 0}-x\right|^{2} \mathrm{~d} s\right] \\
\leq & C \delta \mathbb{E}^{\mathscr{F _ { t }}}\left[\sup _{t \leq s \leq t+\delta}\left|X^{t, x ; v, 0}-x\right|^{2}\right] \\
\leq & \delta^{2} .
\end{aligned}
$$

Hence, it yields that

$$
\begin{aligned}
\left|Y_{t}^{1, v, 0}-Y_{t}^{2, v}\right|= & \mid \mathbb{E}^{\mathscr{F _ { t }}}\left[\int _ { t } ^ { t + \delta } \left(F\left(s, X_{s}^{t, x ; v, 0}, Y_{s}^{1, v, 0}, Z_{s}^{1, v, 0}, v_{s}\right)\right.\right. \\
& \left.-F\left(s, x, Y_{s}^{2, v}, Z_{s}^{2, v}, v_{s}\right)\right) \mathrm{d} s \mid \\
\leq & C \mathbb{E}^{\mathscr{F}_{t}}\left[\int_{t}^{t+\delta}\left|X_{s}^{1, v, 0}-x\right|^{2} \mathrm{~d} s\right] \\
& +C \delta^{\frac{1}{2}} \mathbb{E}^{\mathscr{F} t}\left[\int_{t}^{t+\delta}\left|Y_{s}^{1, v, 0}-Y_{s}^{2, v}\right|^{2} \mathrm{~d} s\right]^{\frac{1}{2}} \\
& +C \delta^{\frac{1}{2}} \mathbb{E}^{\mathscr{F} t}\left[\int_{t}^{t+\delta}\left|Z_{s}^{1, v, 0}-Z_{s}^{2, v}\right|^{2} \mathrm{~d} s\right]^{\frac{1}{2}} \\
\leq & C \delta^{\frac{3}{2}} .
\end{aligned}
$$

We thus complete the proof. 
Acknowledgements. The author highly appreciates the constructive comments and suggestions of the editor Prof. Alberto Bressan and the referee which led to several improvements of the original version. The author also wishes to thank Prof. Xiuqing Chen and Prof. Wulin Suo for their valuable comments and discussions which improved the presentation of this manuscript.

\section{References}

\section{References}

[1] L. Alvarez, A class of solvable singular stochastic control problems, Stochastics Stochastics Rep. 67 (1999) 83-122.

[2] L. Alvarez, Singular stochastic control, linear diffusions, and optimal stopping: A class of solvable problems, SIAM J. Control Optim. 39 (2001) 1697-1710.

[3] V.E. Beněs, L.A. Shepp, H.S. Witzsenhausen, Some solvable stochastic control problems, Stochastics 4 (1980) 39-83.

[4] A. Bensoussan, J. L. Lions, Applications des Inéquations Variationnelles en Contrôle Stochastique, Dunod, Paris, 1978.

[5] M. I. Borodowski, A.S. Bratus, F.L. Chernous'ko, Optimal impulse correction under random perturbation, J. Appl. Math. Mech. 39 (1975) 797-805.

[6] F. Baldursson, Singular stochastic control and optimal stopping, Stochastics 21 (1987) 1-40.

[7] G. Barles, R, Buckdahn and E. Pardoux, Backward stochastic differential equations and integral-partial differential equations, Stochastics Stochastics Rep, Volume 60, (1997) Issue 1-2.

[8] F. Boetius, Bounded variation singular stochastic control and associated Dynkin game, in Mathematical Finance, Trends Math., M. Kohlmann and S. Tang, eds., Birkhäuser, Basel, (2001) 111-120.

[9] F. Boetius, Singular Stochastic Control and Its Relations to Dynkin Game and Entry-Exit Problems, Ph.D. thesis, University of Konstanz, 2001.

[10] F. Boetius and M. Kohlmann, Connections between optimal stopping and singular stochastic control, Stochastic Process. Appl., 77 (1998) 53-281 
[11] J. Bather and H. Chernoff, Sequential decisions in the control of a spaceship, in Proceedings of the Fifth Berkeley Symposium on Mathematical Statistics and Probability, Vol. III: Physical Sciences, University of California Press, Berkeley, CA, (1967) 181-207.

[12] F. Baldursson and I. Karatzas, Irreversible investment and industry equilibrium, Finance Stoch. 1 (1997) 69-89.

[13] R. Buckdahn and J. Li, Stochastic differential games and viscosity solutions of Hamilton-Jacobi-Bellman-Isaacs equations, SIAM J. Control Optim. 47 (2008) 444-475.

[14] R. Buckdahn , B. Labed , C. Rainer, and L. Tamer, Existence of an optimal control for stochastic control systems with nonlinear cost functional, An International Journal of Probability and Stochastic Processes Vol. 82, (2010) Issue 3

[15] K. Bahlali, B. Gherbal and B. Mezerdi, Existence of optimal controls for systems driven by FBSDEs, Systems Control Lett. 60 (2011) 344-349.

[16] V. Barbu, C. Benazzoli, L. Di Persio, Mild solutions to the dynamic programming equation for stochastic optimal control problems, Automatica 93, (2018) 520-526.

[17] A. Cadenillas, U. Haussmann, The stochastic maximum principle for a singular control problem, Stochastics Stochastics Rep Vol 49, (1994) 211-237.

[18] M. B. Chiarolla and U. G. Haussmann, Optimal control of inflation: Central bank problem, SIAM J. Control Optim. 36 (1998) 1099-1132.

[19] P. Chow, J.-L. Menaldi, and M. Robin, Additive control of stochastic linear systems with finite horizon, SIAM J. Control Optim. 23 (1985) 858-899.

[20] M. G. Crandall, H. Ishii, and P.-L. Lions, User's guide to viscosity solutions of second order partial differential equations, Bull. Amer. Math. Soc. (N.S.) 27 (1992) 1-67.

[21] L. Chen, Z. Wu, Dynamic programming principle for stochastic recursive optimal control problem with delayed systems ESAIM-Control, Optimisation and Calculus of Variations 18 (4), (2012) 1005-1026. 
[22] F. Cordoni, L. Di Persio, Stochastic reaction-diffusion equations on net-works with dynamic time-delayed boundary conditions Journal of Mathematical Analysis and Applications 451 (1), (2017) 583-603.

[23] Z. Chen and X. Feng, Backward stochastic differential equations with rankbased data, Science China Mathematics January 2018 Vol. 61 No. 1: (2018) $27-56$.

[24] M. H. A. Davis, A.R. Norman, Portfolio Selection with Transaction Costs, Mathematics of Operations Research Vol. 15, No. 4, (1990) 676-713.

[25] F. Dufour, B. Miller, Singular stochastic control problems, SIAM J. Control Optim. Vol. 43, No. 2, (2004) 708-730.

[26] F. Dufour, B. Miller, Maximum principle for singular stochastic control problems, SIAM J. Control Optim. Vol. 45, No. 2, (2006) 668-698.

[27] K. R. Dahl \& B. Øksendal, Singular recursive utility, Stochastics VOL. 89, NOS. 6-7, (2017) 994-1014.

[28] D. Duffie, L. Epstein, Stochastic differential utility, Econometrica 60 (1992) 353-394.

[29] N. El Karoui and I. Karatzas, Probabilistic aspects of finite-fuel, reflected follower problems, Acta Appl. Math. 11 (1988) 223-258.

[30] N. El Karoui and I. Karatzas, A new approach to the skorohod problem, and its applications, Stochastics Stochastics Rep. 34 (1991) 57-82.

[31] W. Fleming and H. Soner, Controlled Markov Processes and Viscosity Solutions, Springer-Verlag, New York, 1993.

[32] W. H. Fleming, R. W. Rishel, Deterministic and Stochastic Optimal Control, Springer- Verlag, New York, 1975.

[33] G. Fu, U. Horst, Mean Field Games with Singular Controls, SIAM J. Control Optim. Vol. 55, No. 6, (2017) 3833-3868, .

[34] S. Hamadéne and J. P. Lepeltier, Reflected backward stochastic differential equations and mixed game problem, Stochastic Process. Appl. 85 (2000) 177188. 
[35] U. Haussmann, W. Suo, Singuar optimal stochastic controls I: Existence, SIAM J. Control Optim. Vol. 33, No. 3, (1995) 916-936.

[36] U. Haussmann, W. Suo, Singuar optimal stochastic controls II: Dynamic programming. SIAM J. Control Optim. Vol. 33, No. 3, (1995) 937-959.

[37] H. Ishii. Uniqueness of unbounded viscosity solutions of Hamilton-Jacob equations, Indiana U. Math. J. 26 (1984) 721-748.

[38] N. El Karoui, S. Peng, and M. C. Quenez, Backward stochastic differential equations in finance, Math. Finance 7 (1997) 1-71.

[39] I. Karatzas, The monotone follower problem in stochastic decision theory, Appl. Math. Optim. 7 (1981) 175-189.

[40] I. Karatzas, A class of singular control problems, Adv. in Appl. Probab. 15 (1983) 225-254.

[41] I. Karatzas and S. Shreve, Connections between optimal stopping and singular stochastic control I. Monotone follower problems, SIAM J. Control Optim. 22 (1984) 856-877.

[42] I. Karatzas and S. Shreve, Connections between optimal stopping and singular stochastic control II. Reflected follower problems, SIAM J. Control Optim. 23 (1985) 433-451.

[43] I. Karatzas and S. Shreve, Equivalent models for finite-fuel stochastic control, Stochastics 18 (1986) 245-276.

[44] T. Kobila, A class of solvable stochastic investment problems involving singular controls, Stochastics Stochastics Rep 43 (1993) 29-63.

[45] I. Karatzas and H. Wang, A barrier option of American type, Appl. Math. Optim. 42 (2000) 259-279.

[46] N. Krylov, Controlled Diffusion Processes, Springer-Verlag, New York, 1980.

[47] J. Li, S. Peng, Stochastic optimization theory of backward stochastic differential equations with jumps and viscosity solutions of Hamilton-Jacobi-Bellman equations, Nonlinear Analysis 70 (2009) 1776-1796. 
[48] J. Lehoczky and S. Shreve, Absolutely Continuous and Singular Stochastic Control. Stochastics 17, (1986) 91-109.

[49] P. L. Lions, Optimal control of diffusion processes and Hamilton-JacobiBellman equations. Part 1: The dynamic programming principle and applications, and Part 2: Viscosity solutions and uniqueness, Comm. Partial Differential Equations 8, 1101-1174. (1983) 1229-1276.

[50] J. Li , Q. Wei, Optimal control problems of fully coupled FBSDEs and viscosity solutions of Hamilton-Jacobi-Bellman equations SIAM J. Control Optim. 52 (3), (2014) 1622-1662.

[51] J. Ma, On the principle of smooth fit for a class of singular stochastic control problems for diffusion, SIAM J. Control Optim. 30 (1992) 975-999.

[52] J.-L. Menaldi and M. Taksar, Optimal correction problem of a multidimensional stochastic system, Automatica 25 (1989) 223-232.

[53] L. F. Martins and H. J. Kushner, Routing and singular control for queueing networks in heavy traffic, SIAM J. Control Optim. 28 (1990) 1209-1233.

[54] E. Pardoux, S. Peng, Adapted solution of a backward stochastic di-erential equation, Systems Control Lett. 14 (1990) 55-61.

[55] S. Peng, Probabilistic interpretation for systems of quasilinear parabolic partial differential equations, Stochastics Stochastics Rep. 37 (1991) 61-74.

[56] S. Peng, A generalized dynamic programming principle and HJB equation, Stochastics Stochastics Rep. 38 (1992) 119-134.

[57] S. Peng, BSDE and stochastic optimizations, in: J. Yan, S. Peng, S. Fang, L. Wu, Topics in Stochastic Analysis, Science Press, Beijing, 1997 (Chapter 2) (in Chinese).

[58] H. Pagès, D. Possamaï, A mathematical treatment of bank monitoring incentives, Finance and Stochastics 18 (1), (2014) 39-73.

[59] H. M. Soner, S. E. Shreve, Regularity of the value function for a twodimensional singular stochastic control problem, SIAM J. Control Optim. 27 (1989) 876-907. 
[60] S. E. Shreve, J. P. Lehoczky, and D. P. Gaver, Optimal consumption for general diffusions with absorbing and reflecting barriers, SIAM J. Control Optim. 22 (1984) 55-75.

[61] J. Shi, J. Xu, H. Zhang, Stochastic recursive optimal control problem with time delay and applications, Mathematical Control and Related Fields 5 (4), (2015) 859-888.

[62] S. A. Williams, P. L. Chow, J. L. Menaldi, Regularity of the free boundary in singular stochastic control, J. Differential Equations 111 (1994) 175-201.

[63] B. Wang, Singular control of stochastic linear systems with recursive utility, Systems Control Lett. 51 (2004) 105-122.

[64] Z. Wu, Z. Yu, Dynamic programming principle for one kind of stocastic recursive optimal control problem and Hamilton-Jacobi-Bellman equation, SIAM J. Control Optim. Vol. 47, No. 5, (2008) 2616-2641.

[65] J. Yong, X. Y. Zhou, Stochastic Controls. Hamiltonian Systems and HJB Equations, Springer-Verlag, New York, 1999.

[66] H. Zhu, Generalized solution in singular control: The nondegenrate problem, Appl. Math. Optim. 25 (1992) 225-245.

[67] L. Zhang, Stochastic verification theorem of forward-backward controlled systems for viscosity solutions, Systems Control Lett. 61 (2012) 649-654.

[68] L. Zhang, Q. Zhou, Near-optimal control of stochastic recursive systems via viscosity solution, J Optim Theory Appl. 178 (2), (2018) 363-382. 